\title{
Transient effects in oilfield cementing flows: Qualitative behaviour
}

\author{
M. A. MOYERS-GONZÁLEZ1, I. A. FRIGAARD, ${ }^{2}$ O. SCHERZER ${ }^{3}$ \\ and T.-P. TSAI ${ }^{4}$ \\ ${ }^{1}$ Département de mathématiques et de statistique, Université de Montréal, CP 6128 succ. Centre-Ville, \\ Montréal, Quebec, Canada H3C $3 \mathrm{~J} 7$ \\ email: moyers@dms.umontreal.ca \\ ${ }^{2}$ Department of Mathematics and Department of Mechanical Engineering, University of British Columbia, \\ 2054-6250 Applied Science Lane, Vancouver, British Columbia, Canada V6T $1 Z 4$ \\ email: frigaard@mech.ubc.ca \\ ${ }^{3}$ Department of Computer Science, Universität Innsbruck, Technikerstraße 25, A-6020 Innsbruck, Austria \\ email: Otmar.Scherzer@uibk.ac.at \\ ${ }^{4}$ Department of Mathematics, University of British Columbia, 1984 Mathematics Road, Vancouver, \\ British Columbia, Canada V6T $1 Z 2$ \\ email: ttsai@math.ubc.ca
}

(Received 18 July 2006; revised 21 March 2007)

\begin{abstract}
We present an unsteady Hele-Shaw model of the fluid-fluid displacements that take place during primary cementing of an oil well, focusing on the case where one Herschel-Bulkley fluid displaces another along a long uniform section of the annulus. Such unsteady models consist of an advection equation for a fluid concentration field coupled to a third-order nonlinear PDE (Partial differential equation) for the stream function, with a free boundary at the boundary of regions of stagnant fluid. These models, although complex, are necessary for the study of interfacial instability and the effects of flow pulsation, and remain considerably simpler and more efficient than computationally solving three-dimensional Navier-Stokes type models. Using methods from gradient flows, we demonstrate that our unsteady evolution equation for the stream function has a unique solution. The solution is continuous with respect to variations in the model physical data and will decay exponentially to a steady-state distribution if the data do not change with time. In the event that density differences between the fluids are small and that the fluids have a yield stress, then if the flow rate is decreased suddenly to zero, the stream function (hence velocity) decays to zero in a finite time. We verify these decay properties, using a numerical solution. We then use the numerical solution to study the effects of pulsating the flow rate on a typical displacement.
\end{abstract}

\section{Introduction}

Primary cementing is an operation carried out at least once during construction of every oil and gas well. The aim of the operation is to cement a steel casing into the drilled wellbore. The hardened cement both provides structural support for the well and produces an hydraulic seal. The latter prevents migration of formation fluids from one rock stratum to another, which can result in lost productivity as well as having environmental consequences if the fluids leak to surface. The operation proceeds by pumping washes, 
spacer fluids and liquid cement slurries, in sequence, down the inside of the steel casing from surface. At the bottom of the hole, these fluids enter the annulus and displace upwards whatever fluids are in place, typically a drilling mud. Problems arise due to the eccentricity of the annulus and the rheological and physical parameters of the fluids. For example, a fluid with a yield stress is susceptible to becoming stuck on the narrow side of the annulus, bridging the gap between the casing and formation.

In [3, 15-17] we have studied primary cementing displacements along an eccentric annulus, using a Hele-Shaw type model. Although, in general, a sequence of fluids is pumped along the annulus, each fluid displacing the one in front, the fluid volumes pumped are relatively large and the annular geometry changes slowly in the axial direction. Therefore, the essential dynamics of the displacement may be studied by considering what happens between any two fluids on an annular section of constant geometry. In [3, 15-17] this simpler situation has been modelled by the following two-dimensional elliptic PDE, in azimuthal and axial spatial directions:

$$
\begin{aligned}
\nabla \cdot\left[\mathbf{S}_{\mathrm{s}}+\mathbf{f}\right] & =0, \\
\mathbf{S}_{\mathrm{s}}=\left[\frac{\chi\left(\left|\nabla \Psi_{\mathrm{s}}\right|\right)+\tau_{Y} / H}{\left|\nabla \Psi_{\mathrm{s}}\right|}\right] \nabla \Psi_{\mathrm{s}} & \Longleftrightarrow\left|S_{\mathrm{s}}\right|>\frac{\tau_{Y}}{H}, \\
\left|\nabla \Psi_{\mathrm{s}}\right|=0 & \Longleftrightarrow\left|S_{\mathrm{s}}\right| \leqslant \frac{\tau_{Y}}{H} .
\end{aligned}
$$

Here $\Psi_{\mathrm{s}}$ is the stream-function, the unwrapped narrow annular space is $(\phi, \xi) \in(0,1) \times$ $(0, Z)$, the annular gap half-width is $H(\phi)=1+e \cos \pi \phi$, and $e \in[0,1)$ is the eccentricity (see Figure 1). The function $\chi$ is a positive increasing function of $\left|\nabla \Psi_{\mathrm{s}}\right|$, which represents the viscous part of frictional pressure gradient. The exact form of $\chi$ depends on the local width of the annular gap, $H$, and on the rheological parameters that characterise the fluid; $\tau_{Y}$ is the fluid yield stress. Typical functions $\chi$ are plotted in Figure 2 for a Herschel-Bulkley over a range of parameters. The vectorfield $\mathbf{f}$, which represents the buoyancy forces, and the rheological properties of the fluids may depend on time and space via the mixture concentrations, which in the case of two fluids can be characterised by the concentration of fluid 1 , say $\bar{c}(\phi, \xi, t)$. In [3] the concentration is simply advected along the annulus:

$$
\frac{1}{\epsilon} \frac{\partial}{\partial t}[H \bar{c}]+\frac{\partial}{\partial \phi}\left[H \bar{v}_{\mathrm{s}} \bar{c}\right]+\frac{\partial}{\partial \xi}\left[H \bar{w}_{\mathrm{s}} \bar{c}\right]=0,
$$

where $\epsilon$ denotes a timescale ratio (defined later), and the gap-averaged velocities are defined in terms of the stream function by the following:

$$
\bar{v}_{\mathrm{s}}=-\frac{1}{H} \frac{\partial \Psi_{\mathrm{s}}}{\partial \xi}, \quad \bar{w}_{\mathrm{s}}=\frac{1}{H} \frac{\partial \Psi_{\mathrm{s}}}{\partial \phi} .
$$

The system (1.1)-(1.5), which is essentially a Hele-Shaw model, has proven itself useful for understanding many features of the primary cementing operation. For example, we are able to identify the important case when there is a steady displacement front that advances as a travelling wave along the well. For some parameter ranges we are even able to provide an analytical description of the steady-state shape. If there is no steadily 

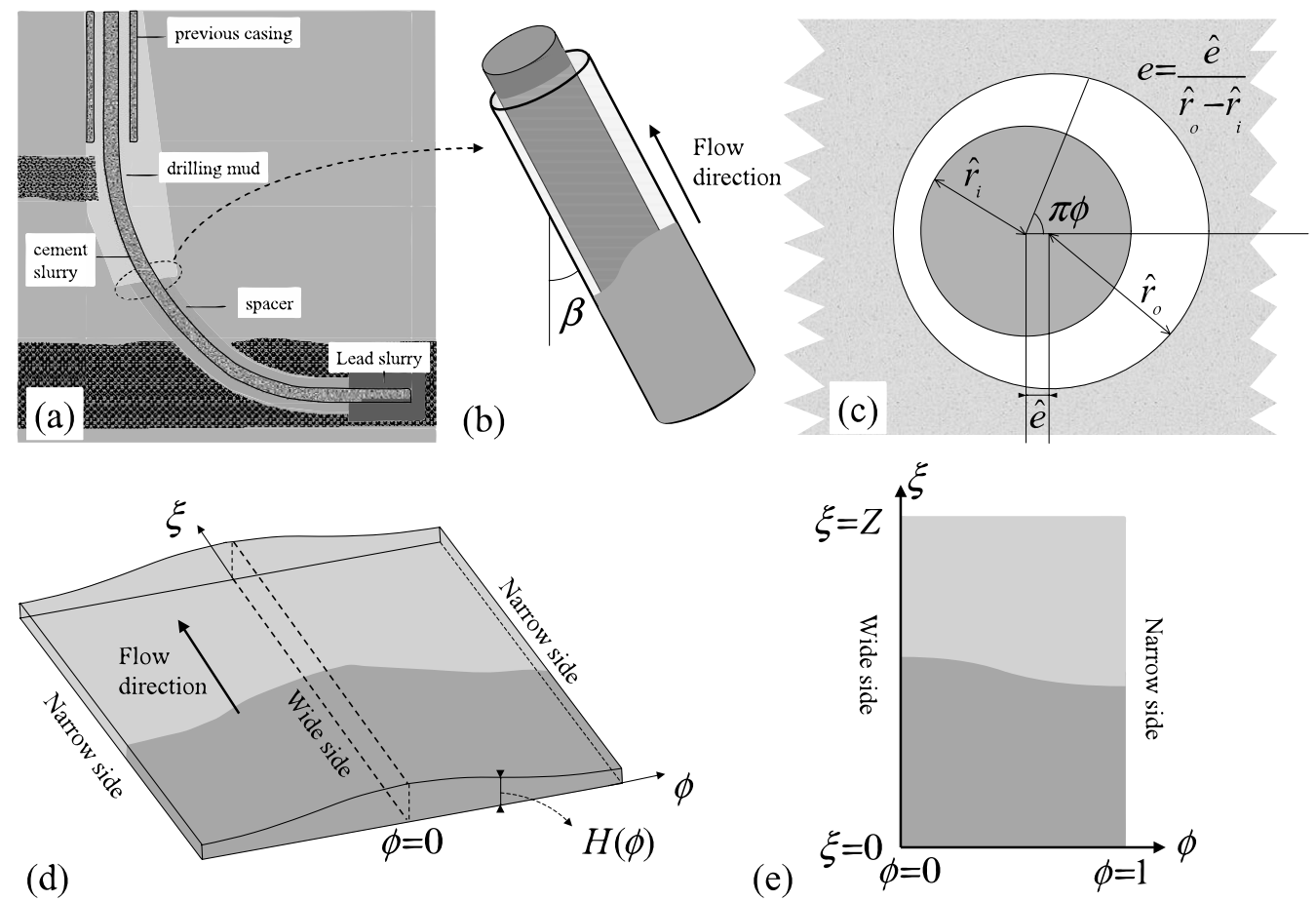

FIgURE 1. Cementing geometries: (a) schematic of fluid stages pumped during a typical primary cementing displacement; (b) uniform section of eccentric annulus; (c) eccentric annular cross-section; (d) periodic eccentric annular Hele-Shaw cell; (e) final computational domain, assuming symmetry at wide and narrow sides of the annulus.
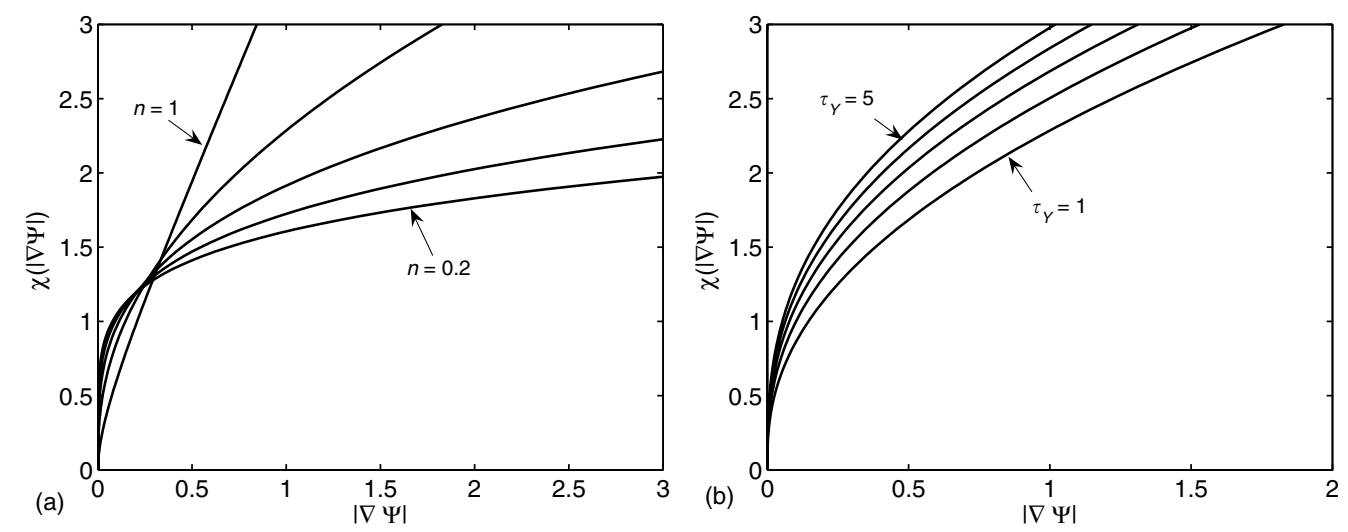

Figure 2. Examples of the function $\chi(|\nabla \Psi|)$ for a Herschel-Bulkley fluid: (a) $H=1, \tau_{Y}=1, \kappa=1$, $n=1,1 / 2,1 / 3,1 / 4,1 / 5$; (b) $H=1, \tau_{Y}=1,2,3,4,5, \kappa=1, n=1 / 2$.

advancing front, we can identify two possibilities: (i) an unsteady displacement front advances along the wide side of the annulus faster than along the narrow side and (ii) the displaced fluid becomes stuck on the narrow side of the annulus. These results and others 
are described in the sequence of papers [3, 15-17]. The focus of this paper is on a two-dimensional time-dependent extension of (1.1), which we define and derive below, in Section 2.

There are a number of practical situations that warrant consideration of a transient model for $\Psi$. First, pulsation techniques have been advocated at different times for primary cementing, e.g. [5], with different claimed benefits. To pulse the flow rate is possible with the steady model (1.1), via the boundary conditions. However, since time dependency enters $\Psi_{\mathrm{s}}$ only via the concentration and boundary conditions, the pulsation appears to simply superimpose an axial oscillation on the flow, i.e. the coupling is in one direction only. To study, for example, whether pulsation can aid in the removal of static mud channels that form on the narrow side of the eccentric annulus, it appears necessary to allow transient evolution of the stream-function.

Secondly as shown in [17], for certain rheological combinations an unsteady displacement front evolves that advances up the wide side of the annulus faster than up the narrow side, with the interface eventually becoming pseudo-parallel to the annulus axis. Although this situation is believed to be very bad for the displacement process, such an assessment may be premature. For example, Tehrani et al. report observing interfacial instabilities for some of these situations [18]. If these interfacial instabilities lead to azimuthal mixing around the annulus, then in fact the displacement may still be effective. In place of unsteady fingering, we will have axial dispersion, which may grow at an acceptably slow rate, e.g. $\sim t^{1 / 2}$ rather than $\sim t$. Thus, the study of interfacial instabilities in parallel multi-layer annular Hele-Shaw flows is a problem of considerable practical interest, and requires a transient model in place of (1.1). This is the subject of a companion paper [14].

In this paper we focus on derivation of a well-posed transient extension of (1.1), and its application to the problem of flow pulsation during primary cementing. A brief outline of our paper is as follows. The model derivation is dealt with in Section 2 below, terminating with the classical formulation of the unsteady Hele-Shaw model for the stream function. Section 3 contains the key mathematical results of the paper. After casting the problem in a functional analytical setting, we show that there exists a unique solution to both the steady and transient problems. Various continuity results are given, and we also examine decay of the transient solution to a steady state, with and without an imposed flow rate. Much of the detail of these results is confined to [13]. Computational results are presented in Section 4. We test our numerical algorithm against the decay results and then apply the algorithm to the study of pulsatile flows. The paper concludes with a short discussion in Section 5.

\section{Modelling transient bulk flow cementing displacements}

The aim of our modelling is to allow for the possibility of coupled temporal effects between the velocity field and fluid concentration field, whilst retaining the relative simplicity of the Hele-Shaw approach. In [3], time derivatives in the momentum balance were neglected by considering the limit of a short viscous timescale, $\hat{t}_{\mathrm{v}}$, relative to the advective timescale, $\hat{t}_{\mathrm{a}}$. Here these time derivatives are retained, but the modelling approach is otherwise similar to that of [3], to which the reader is referred for details; see also [13]. Below we give only a brief overview of the derivation. 
Table 1. Notation for scaled variables, velocities and domain

\begin{tabular}{cccc}
\hline \hline Independent variable & Scaled variables & Velocity & Range \\
\hline Radial & $y$ & $u$ & {$[0, H(\phi)]$} \\
Azimuthal & $\phi$ & $v$ & {$[0,1]$} \\
Axial & $\xi$ & $w$ & {$[0, Z]$} \\
\hline \hline
\end{tabular}

When a steel casing is inserted into a borehole, the annulus geometry typically satisfies

[mean annular gap] $\ll[$ mean circumference] $\ll[$ length of annulus $]$.

Here we consider only a short section of uniform eccentric annulus (part of the well or a laboratory experiment), and assume that fluid $1(\bar{c}=1)$ displaces fluid $2(\bar{c}=0)$ upwards along the annulus. The section of the annulus is assumed long enough so that intermediate concentrations are found only in the interior.

\subsection{Reduced shear flow model}

We follow the classical Hele-Shaw approach. Velocities in azimuthal and axial directions are scaled with the mean flow velocity $\hat{w}_{\mathrm{a}}$, derived from a representative imposed flow rate. Lengths in these directions are scaled with the half-circumference, $\pi \hat{r}_{\mathrm{a}}$, where $\hat{r}_{\mathrm{a}}=\left(\hat{r}_{o}+\hat{r}_{i}\right) / 2$ is the mean radius. The annulus is eccentric, with eccentricity $e$, and is inclined at angle $\beta$ to the vertical (see Figure 1). Only half of the annulus circumference is considered. The ratio of mean half-gap to mean radius is denoted $\delta=\left(\hat{r}_{o}-\hat{r}_{i}\right) /\left(\hat{r}_{o}+\hat{r}_{i}\right)$, and the Hele-Shaw approach requires that $\delta / \pi \ll 1$, i.e. this is a narrow annulus. The radial distance from the centreline of the annulus to the walls is scaled with $\delta \hat{r}_{\mathrm{a}}$, and the radial velocity accordingly to preserve continuity. Stresses and pressure scales are chosen to balance the maximal shear-stresses in the usual fashion; see [3]. Time is scaled with $\hat{t}_{\mathrm{v}}$. In this way, it is straightforward to derive the following dimensionless reduced shear flow model,

$$
\begin{aligned}
\rho \frac{\partial v}{\partial t} & =-\frac{\partial p}{\partial \phi}+\frac{\partial}{\partial y} \tau_{\phi y}+g_{\phi}, \\
\rho \frac{\partial w}{\partial t} & =-\frac{\partial p}{\partial \xi}+\frac{\partial}{\partial y} \tau_{\xi y}+g_{\xi} \\
\frac{\partial p}{\partial y} & =0
\end{aligned}
$$

in which all quantities are $O(1)$. Here $\rho$ is the fluid density, $(v, w)$ are the azimuthal and axial velocity components, $p$ is the pressure and $\left(g_{\phi}, g_{\xi}\right)$ denotes the scaled gravitational acceleration vector. The azimuthal coordinate is $\phi \in[0,1]$, where $\phi=0$ denotes the wide (upper) side of the annulus and $\phi=1$ denotes the narrow (lower) side of the annulus. The axial coordinate is $\xi \in[0, Z]$. Table 1 gives the ranges of the scaled independent variables, the velocities and their domain. As usual, only the principal shear stresses $\left(\tau_{\phi y}, \tau_{\xi y}\right)$ are retained, and the constitutive relations are discussed below in Section 2.2. The scaled 
radial coordinate is $y$ and the annulus walls are denoted by $y=\mp H(\phi)$, where

$$
H(\phi)=1+e \cos \pi \phi .
$$

Because the annulus is relatively narrow and the walls are stationary, we make the simplifying assumption that the velocity $(v, w)$ is symmetric about the centreline $y=0$, and thus consider only $y \in[0, H]$. Coupled to $(2.1)-(2.3)$ is the leading-order continuity equation:

$$
0=\frac{\partial u}{\partial y}+\frac{\partial v}{\partial \phi}+\frac{\partial w}{\partial \xi} .
$$

\subsection{Rheological assumptions}

The fluids used in cementing are shear-thinning generalised Newtonian fluids, often with a yield stress. After the Hele-Shaw scaling, the principal components of the rate of strain are $^{1} \dot{\gamma}_{\phi y} \sim v_{y}$ and $\dot{\gamma}_{\xi y} \sim w_{y}$. Thus at the leading order, the second invariant of the rate of strain $\dot{\gamma}$ is given by $\dot{\gamma}=\left[v_{y}^{2}+w_{y}^{2}\right]^{1 / 2}$. For generalised Newtonian fluids the leading-order shear stresses and rates of strain are related by a law of form:

$$
\tau_{i j}=\eta(\dot{\gamma}) \dot{\gamma}_{i j}, \quad i j=\phi y, \quad \xi y,
$$

where $\eta$ is referred to as the effective viscosity. When the fluid has a yield stress, $\eta(\dot{\gamma}) \rightarrow \infty$ as $\dot{\gamma} \rightarrow 0$, and $\tau_{i j}$ becomes indeterminate. In [3,15-17] we have assumed that the fluids are characterisable as Herschel-Bulkley fluids, which are parameterised by three parameters: $\kappa, n$ and $\tau_{Y}$, which are the consistency, power-law index and yield stress of the fluid, respectively. Here we extend the Herschel-Bulkley model slightly, by addition of a highshear viscosity, $\mu_{\infty}$, and work with the constitutive law

$$
\begin{array}{rlrl}
\tau_{i j} & =\left[\mu_{\infty}+\kappa \dot{\gamma}^{n-1}+\frac{\tau_{Y}}{\dot{\gamma}}\right] \dot{\gamma}_{i j}, & & \tau>\tau_{Y}, \\
\dot{\gamma}=0, & & \tau \leqslant \tau_{Y} .
\end{array}
$$

In practice, we shall suppose that $\mu_{\infty}$ is small. Since the fluids are shear-thinning, we consider $0<n \leqslant 1$. Lastly, we note that for computational purposes, the fluid properties $\kappa, n, \tau_{Y}$ and $\mu_{\infty}$ must be specified as functions of the concentration $\bar{c}$, and as functions of the physical properties of the pure fluids 1 and 2 .

There are two motivations for this modified rheological model. First from the physical perspective, the Herschel-Bulkley model (set $\mu_{\infty}=0$ above) implies an effective viscosity: $\eta(\dot{\gamma})=\kappa \dot{\gamma}^{n-1}+\tau_{Y} / \dot{\gamma} \rightarrow 0$, as $\dot{\gamma} \rightarrow \infty$. This is unrealistic, since suspension viscosities commonly approach a Newtonian plateau for large $\dot{\gamma}$, i.e. the Herschel-Bulkley model is really intended to model low-shear behaviour. The second motivation is mathematical. Without

1 For fluids with a yield stress, this statement needs modifying. Within the psuedo-plug region at the channel centre (where the leading-order shear stresses nominally vanish) the extensional stresses are of the same order as the shear stresses. However, both rates of strain are of first order in the aspect ratio, i.e. small, and therefore this does not affect computation of the gap-averaged velocities, at leading order. See [17] for a more thorough discussion of this and the effects of the so-called lubrication paradox in the context of our model. 
the high-shear viscosity we will later be forced to work in subspaces of $L^{1+n}$ and $W^{1,1+n}$, rather than $L^{2}$ and $H^{1}$. Although most of what we prove in the Hilbert space setting can be extended to $L^{1+n}$ and $W^{1,1+n}$, it is less convenient and involves additional analysis. From the numerical perspective this is anyway unnecessary, since numerically we end up working in finite-dimensional subspaces, which lie in both $\left\{L^{1+n}, W^{1,1+n}\right\}$ and in $\left\{L^{2}, H^{1}\right\}$.

\subsection{Transient Hele-Shaw model derivation}

We first integrate (2.4) across the annular gap, using no-slip boundary conditions at the annulus walls, to eliminate the radial velocity $u$ :

$$
0=\frac{\partial}{\partial \phi}[H \bar{v}]+\frac{\partial}{\partial \xi}[H \bar{w}],
$$

(recall that the overbar denotes a quantity averaged across the annular gap). This prompts definition of a stream function $\Psi(\phi, \xi, t)$, for the gap-averaged flow:

$$
H \bar{w}=\frac{\partial \Psi}{\partial \phi}, \quad H \bar{v}=-\frac{\partial \Psi}{\partial \xi} .
$$

As is usual in the Hele-Shaw approach, we would like to derive a closure of the system (2.1) and (2.2), in terms of either the stream function or the pressure. In [3, 15-17] we have chosen the stream function as the base variable, since the pressure gradient is indeterminate in areas of the annulus where the yield stress is not exceeded, and hence adopt the same choice here.

We may observe that averaging (2.1) and (2.2) across the annular gap does not lead to a closure in terms of the stream function. The reduced shear flow model is properly three-dimensional. Although it is possible to still work with this model, and solution is certainly faster than solving the Navier-Stokes equations, we have lost the advantage of two dimensionality. One way to resolve this is to replace the time derivatives in (2.1) and (2.2) with the time derivatives of the gap-averaged velocities, i.e. we consider the system:

$$
\begin{aligned}
\rho \frac{\partial \bar{v}}{\partial t} & =-\frac{\partial p}{\partial \phi}+\frac{\partial}{\partial y} \tau_{\phi y}+g_{\phi}, \\
\rho \frac{\partial \bar{w}}{\partial t} & =-\frac{\partial p}{\partial \xi}+\frac{\partial}{\partial y} \tau_{\xi y}+g_{\xi} .
\end{aligned}
$$

In doing this, we essentially commute the spatial averaging and time-derivative operators. In [13] we show that the $L^{1}$ norm of the velocity solutions of (2.8) and (2.9) (evaluated across the annular gap) remains close to that of (2.1) and (2.2), provided that the data change sufficiently slowly. ${ }^{2}$ As we want to work anyway with the gap-averaged velocities, this is sufficient for our needs.

2 The approach in [13] involves considering the variational problems related to solution of (2.8) and (2.9) and comparing with the variational problem that stems from (2.1) and (2.2). Essentially, if the data change sufficiently slowly, both of these problems decay to the same pseudo-steady solution. We show that if the data changes are uniformly $O(\epsilon)$ over a slow timescale, we can expect that $\|\overline{\boldsymbol{u}}-\boldsymbol{u}\|_{L^{1}}(t)=O(\epsilon)$ also. Physically, this means that provided the flow has sufficient time for viscosity to act, before the fluid properties or any other process features change locally, then the derivative of the average is close to the average of the derivative. Evidently, in the case that we have 
Turning now to the system (2.8) and (2.9), the acceleration, pressure and gravitational terms do not vary with $y$. The gravitational acceleration terms are

$$
\left(g_{\phi}, g_{\xi}\right)=\left(\frac{\rho \sin \beta \sin \pi \phi}{S t^{*}},-\frac{\rho \cos \beta}{S t^{*}}\right),
$$

where $S t^{*}$ is the Stokes number, defined below in Section 2.3.3. Integrating (2.8) and (2.9) from zero to $y$ and using the symmetry condition $(\tau=0$ at $y=0)$, we have

$$
\tau(y, \phi, \xi, t)=-\left(\boldsymbol{G}-\rho \overline{\boldsymbol{u}}_{t}\right) y .
$$

Note that the shear stress is linear in $y$. The position in the annular gap where $|\tau|=\tau_{Y}$ is called the yield surface, and separates the fluid layer into yielded $\left(|\tau|>\tau_{Y}\right)$ and unyielded $\left(\tau_{Y} / A<y \leqslant H\right)$ regions.

Using (2.6), we may uniquely define the strain rate for a given shear stress $|\tau|$, and thus define the effective viscosity via the relation

$$
|\tau|=\eta(\dot{\gamma}) \dot{\gamma} \Rightarrow \dot{\gamma}=|\tau| / \eta(\dot{\gamma})
$$

From (2.6) we may see that as $|\tau| \rightarrow \tau_{Y}$ (which occurs as $y \rightarrow \tau_{Y} / A$ ), $\eta(\dot{\gamma}) \rightarrow \infty$. Using $\dot{\gamma}$ to define the velocity gradient, and integrating with respect to $y$ (using also (2.11) and (2.6)), we have the velocity:

$$
\boldsymbol{u}(y, \phi, \xi, t)=\left[\boldsymbol{G}-\rho \overline{\boldsymbol{u}}_{t}\right] \int_{y}^{H} \frac{\tilde{y}}{\eta(\dot{\gamma}(\tilde{y}))} \mathrm{d} \tilde{y},
$$

where $\boldsymbol{u}=(v, w)$ and

$$
\boldsymbol{G}=\left(-\frac{\partial p}{\partial \phi}+\frac{\rho \sin \beta \sin \pi \phi}{S t^{*}},-\frac{\partial p}{\partial \xi}-\frac{\rho \cos \beta}{S t^{*}}\right) .
$$

Note that the integral in the right-hand side of (2.12) is defined for all $y \in[0, H]$, but since $\eta(\dot{\gamma}) \rightarrow \infty$ as $y \rightarrow \tau_{Y} / A$, the integrand will have zero contribution within the unyielded fluid layer.

We see that $\boldsymbol{u}$ is instantaneously parallel to $\boldsymbol{G}-\rho \overline{\boldsymbol{u}}_{t}$. Writing $s(y)=|\boldsymbol{u}|(y)$, at each fixed $(\phi, \xi, t)$, the speed $s$ is related to the modified pressure gradient, $A$, via the one-dimensional shear flow problem:

$$
-A=\frac{\mathrm{d}}{\mathrm{d} y}\left[\eta\left(\left|\frac{\mathrm{d} s}{\mathrm{~d} y}\right|\right) \frac{\mathrm{d} s}{\mathrm{~d} y}\right] .
$$

For generalised Newtonian fluids, this problem is straightforwardly solved, either analytically, or numerically by simple quadrature. However, we are interested in the gap-averaged

large variations in the data over a faster timescale, we would not expect any convergence. We note in passing that a similar interchange of averaging and derivative operations is commonly carried out in hydraulics problems (usually to model inertial effects), with the (pragmatic) introduction of a correction factor. 
speed:

$$
\begin{aligned}
|\nabla \Psi| & =H \bar{s}=\int_{0}^{H} s(y) \mathrm{d} y=-\int_{0}^{H} y \frac{\mathrm{d} s}{\mathrm{~d} y}(y) \mathrm{d} y, \\
& =\int_{0}^{H} y \dot{\gamma}(y) \mathrm{d} y=\frac{1}{A^{2}} \int_{\tau_{Y}}^{A H} \tau \dot{\gamma}(\tau) \mathrm{d} \tau,
\end{aligned}
$$

where from (2.6), for $\tau \geqslant \tau_{Y}$,

$$
\tau(\dot{\gamma})=\mu_{\infty} \dot{\gamma}+\kappa \dot{\gamma}^{n}+\tau_{Y}
$$

and $\dot{\gamma}(\tau)$ is obtained by inverting this monotone function. We may observe that $\dot{\gamma}(\tau)$ is a strictly monotone $C^{\infty}$ function of $\tau>\tau_{Y}$. If $n<1$, then as $\tau \rightarrow \tau_{Y}, \dot{\gamma}(\tau) \rightarrow 0$ and the Herschel-Bulkley term dominates: $\dot{\gamma} \sim\left[\left(\tau-\tau_{Y}\right) / \kappa\right]^{1 / n}$. As $\tau \rightarrow \infty$, the high-shear viscous term dominates: $\dot{\gamma} \sim\left(\tau-\tau_{Y}\right) / \mu_{\infty}$. In between these limits, numerical inversion is needed to define $\dot{\gamma}(\tau)$.

The expression (2.14) defines the closure relationship between the gap-averaged flow rate $|\nabla \Psi|$ and the modified pressure gradient $A$. Evidently, as $A \rightarrow \tau_{Y} / H$ the range of the integral vanishes and $|\nabla \Psi| \rightarrow 0$. Since the integrand, $\tau \dot{\gamma}(\tau)$, is strictly positive, increasing faster than linear, and is $C^{\infty}$, for $\tau>\tau_{Y} / H$ we see that $|\nabla \Psi|(A)$ is also $C^{\infty}$ and increases strictly monotonically, for $A>\tau_{Y} / H$. Inverting this relation, we may write $A=A(|\nabla \Psi|)$. For $|\nabla \Psi|>0$ we have that $A\left(|\nabla \Psi|\right.$ ) is strictly positive (bounded strictly below by $\tau_{Y} / H$ ), and strictly monotone. It is convenient to separate the yield stress effects. Below, we shall write

$$
A(|\nabla \Psi|)=\chi(|\nabla \Psi|)+\frac{\tau_{Y}}{H}
$$

Thus, $\chi(|\nabla \Psi|)$ represents the part of the modified pressure gradient surplus to that required to overcome the yield stress locally. We may rewrite $(2.14)$ as

$$
|\nabla \Psi|=\frac{1}{\left[\chi+\tau_{Y} / H\right]^{2}} \int_{\tau_{Y}}^{H \chi+\tau_{Y}} \tau \dot{\gamma}(\tau) \mathrm{d} \tau .
$$

Although we have focused on the relation between $|\nabla \Psi|$ and $A$ (equivalently $\chi$ ), we note that via the constitutive law and the limits on the integration in (2.14) and (2.16), we have the following parametric dependency of these functions:

$$
|\nabla \Psi|=|\nabla \Psi|\left(\chi ; H, \tau_{Y}, \kappa, n, \mu_{\infty}\right), \quad \chi=\chi\left(|\nabla \Psi| ; H, \tau_{Y}, \kappa, n, \mu_{\infty}\right) .
$$

Returning to (2.12), we average across the half-gap, $y \in[0, H]$, to give

$$
\overline{\boldsymbol{u}}=\left[\boldsymbol{G}-\rho \overline{\boldsymbol{u}}_{t}\right] \frac{1}{H} \int_{0}^{H} \int_{y}^{H} \frac{\tilde{y}}{\eta(\tilde{y})} \mathrm{d} \tilde{y} \mathrm{~d} y,
$$

from which we see that $\overline{\boldsymbol{u}}$ is also instantaneously parallel to the vector $\left[\boldsymbol{G}-\rho \overline{\boldsymbol{u}}_{t}\right]$ :

$$
\frac{\left(-\frac{\partial \Psi}{\partial \xi}, \frac{\partial \Psi}{\partial \phi}\right)}{|\nabla \Psi|}=\frac{\left(\frac{\rho}{H} \frac{\partial^{2} \Psi}{\partial \xi \partial t}-\frac{\partial p}{\partial \phi}+g_{\phi},-\frac{\rho}{H} \frac{\partial^{2} \Psi}{\partial \phi \partial t}-\frac{\partial p}{\partial \xi}+g_{\xi}\right)}{A}
$$


For $|\nabla \Psi|>0$, replacing $A$ with $\chi+\tau_{Y} / H$, this implies that

$$
\begin{gathered}
\frac{\rho \partial^{2} \Psi}{\partial \xi \partial t}-\frac{\partial p}{\partial \phi}+\frac{\rho \sin \beta \sin \pi \phi}{S t^{*}}=-\left(\frac{\chi(|\nabla \Psi|)+\tau_{Y} / H}{|\nabla \Psi|}\right) \frac{\partial \Psi}{\partial \xi}, \\
-\frac{\rho}{H} \frac{\partial^{2} \Psi}{\partial \phi \partial t}-\frac{\partial p}{\partial \xi}-\frac{\rho \cos \beta}{S t^{*}}=\left(\frac{\chi(|\nabla \Psi|)+\tau_{Y} / H}{|\nabla \Psi|}\right) \frac{\partial \Psi}{\partial \phi} .
\end{gathered}
$$

Cross-differentiating to eliminate the pressure, we finally arrive at

$$
\nabla \cdot\left[\frac{\rho}{H} \nabla \Psi_{t}\right]=-\nabla \cdot\left[\left(\frac{\chi(|\nabla \Psi|)+\tau_{Y} / H}{|\nabla \Psi|}\right) \nabla \Psi+\mathbf{f}\right],
$$

where $\mathbf{f}$ contains the buoyancy terms:

$$
\mathbf{f}=\left(\rho(\bar{c}) \frac{\cos \beta}{S t^{*}}, \rho(\bar{c}) \frac{\sin \beta \sin \pi \phi}{S t^{*}}\right) .
$$

If $|\nabla \Psi|=0$, we may still cross-differentiate to eliminate the pressure, except that the right-hand side of this system is multi-valued. Thus, we may write

$$
\nabla \cdot\left[\frac{\rho}{H} \nabla \Psi_{t}\right]=-\nabla \cdot[\mathbf{S}+\mathbf{f}]
$$

where

$$
\begin{array}{r}
\mathbf{S}=\left(\frac{\chi(|\nabla \Psi|)+\tau_{Y} / H}{|\nabla \Psi|}\right) \nabla \Psi \Leftrightarrow|\mathbf{S}|>\tau_{Y} / H, \\
|\nabla \Psi|=0 \Leftrightarrow|\mathbf{S}| \leqslant \tau_{Y} / H .
\end{array}
$$

We note also that

$$
\mathbf{S}=\left(-\frac{\rho}{H} \frac{\partial^{2} \Psi}{\partial \phi \partial t}-\frac{\partial p}{\partial \xi}-\frac{\rho \cos \beta}{S t^{*}},-\frac{\rho}{H} \frac{\partial^{2} \Psi}{\partial \xi \partial t}+\frac{\partial p}{\partial \phi}-\frac{\rho \sin \beta \sin \pi \phi}{S t^{*}}\right)
$$

Equation (2.21) is the classical formulation of our evolution problem for $\Psi$ (effectively giving the gap-averaged velocity in the annulus). We shall consider this in a more rigorous setting below in Section 3.

\subsubsection{Boundary conditions}

Equation (2.21) is supplemented with the following boundary conditions:

$$
\begin{gathered}
\Psi(0, \xi, t)=0, \\
\Psi(1, \xi, t)=Q(t),
\end{gathered}
$$

on the wide $(\phi=0)$ and narrow $(\phi=1)$ sides of the annulus, respectively. Here $Q(t)=O_{\mathrm{s}}(1)$ represents the total flow rate through the annulus, appropriately scaled. In a typical well with an inclined borehole, the heavy steel casing lies eccentrically towards the lower side of the hole. Thus, the wide side is taken as the upper side of the annular section, with each 
annular section being symmetric about the (vertical) direction of gravity. As we consider slow flows, we assume that the flow in each half of the annulus is symmetric and for simplicity model only half the annulus, see [3]. Boundary conditions (2.25) and (2.26) fix the flow rate through the annulus and assure that azimuthal velocity is zero on wide and narrow sides, as corresponds to the supposed symmetry of the solution.

Conditions at the ends of the annulus are harder to specify, and depend largely on the situation that we are modelling. In general, we shall suppose that large variations in the fluid concentration occur away from the ends, i.e. we are interested in displacement phenomena away from the ends. Thus, if we consider that the concentration does not change close to the ends of the annulus the flow is parallel and the azimuthal component of the velocity is zero. If we consider our constant geometry section to be a section of the well, then appropriate conditions are

$$
\begin{gathered}
S_{\xi}=0 \Rightarrow \frac{\partial \Psi}{\partial \xi}(\phi, 0, t)=0 . \\
S_{\xi}=0 \Rightarrow \frac{\partial \Psi}{\partial \xi}(\phi, Z, t)=0,
\end{gathered}
$$

since the axial flow of a single fluid along a duct will have parallel streamlines. Alternatively, if we model a lab-scale pilot experiment, we may impose, e.g. the uniform inflow condition

$$
\Psi(\phi, 0, t)=Q(t),
$$

in place of (2.27), retaining the outflow condition (2.28). Finally, if we consider $Z \gg 1$ so that the flow close to the ends of the annulus is far from any concentration variations, we may calculate appropriate one-dimensional flows at the ends, which correspond to stream functions: $\Psi_{\text {in }}(\phi)$ at $\xi=0$, and $\Psi_{\text {out }}(\phi)$ at $z=Z$, respectively. We might then impose

$$
\begin{gathered}
\Psi(\phi, 0, t)=\Psi_{\text {in }}(\phi, t), \\
\Psi(\phi, Z, t)=\Psi_{\text {out }}(\phi, t),
\end{gathered}
$$

in place of (2.27) and (2.28). The Dirichlet conditions (2.30) and (2.31) are easiest to handle analytically, and we assume this below unless otherwise stated.

\subsubsection{Evolution of the fluid concentration}

The gap-averaged fluid concentration evolves according to

$$
\frac{1}{\epsilon} \frac{\partial}{\partial t}[H \bar{c}]+\frac{\partial}{\partial \phi}\left[H \bar{v}_{\mathrm{s}} \bar{c}\right]+\frac{\partial}{\partial \xi}\left[H \bar{w}_{\mathrm{s}} \bar{c}\right]=0,
$$

which is derived exactly as in [3]. At $\xi=0$, the inflow boundary condition is $\bar{c}=1$.

\subsubsection{Dimensionless parameters}

Apart from the $O(1)$ dimensionless fluid properties, there are four dimensionless parameters that govern the model: the eccentricity $e$, the inclination angle $\beta$, the timescale ratio $\epsilon$ 
and the Stokes number $S t^{*}$. The first two are defined in Figure 1. The timescale ratio is defined as $\epsilon=\hat{t}_{\mathrm{v}} / \hat{t}_{\mathrm{a}}$, where the viscous and advective timescales are defined as

$$
\hat{t}_{\mathrm{v}}=\frac{\hat{\rho}^{*}\left(\delta \pi \hat{r}_{\mathrm{a}}\right)^{2}}{\hat{\mu}^{*}}, \quad \hat{t}_{\mathrm{a}}=\frac{\pi \hat{r}_{\mathrm{a}}}{\hat{w}_{\mathrm{a}}} ;
$$

$\hat{\rho}^{*}$ and $\hat{\mu}^{*}$ are density and viscosity scales, respectively. When the viscous timescale is relatively short, we may rescale time with $\epsilon$ and take $\epsilon \rightarrow 0$ to recover the steady model of [3]. For a given fluid pair, $\epsilon$ is sensitive to flow rate changes: as the flow rate increases, $\hat{t}_{\mathrm{a}}$ decreases and $\hat{t}_{\mathrm{v}}$ increases, thus $\epsilon$ increases. Formally, the model in [3] is a model valid at low flow rates. Here we do not necessarily assume $\epsilon \ll 1$. The Stokes number is defined by

$$
S t^{*}=\frac{\hat{\mu}^{*} \hat{w}_{\mathrm{a}}}{\hat{\rho}^{*} \hat{g}\left[\hat{r}_{\mathrm{a}} \delta\right]^{2}}
$$

and represents the ratio of viscous to buoyancy forces, over the scale of the annular gap ( $\hat{g}$ is the gravitational acceleration). Typically, $S t^{*}<1$, say in the range $S t^{*} \in[0.02,0.5]$.

\section{Existence and uniqueness of $\Psi$}

Our full transient displacement model is given by (2.21)-(2.23), with boundary conditions (2.25), (2.26), (2.30) and (2.31), coupled to the concentration evolution equation (2.32). Our focus in this section is to establish that this model is in fact well-posed. We observe that equation (2.21) is not well-defined everywhere, as there may be regions of immobile fluid governed by the transition between (2.22) and (2.23). Thus, in essence we have a free boundary as part of the evolution problem for $\Psi$. As (2.32) represents pure advection, it is clear that the main difficulty with our model is in the problem for $\Psi$, equations (2.21)-(2.23). For this section we therefore ignore (2.32), and ask whether the evolution problem for $\Psi$ is well-posed for a given fixed concentration field (i.e. formally we consider the limit $\epsilon \rightarrow 0$ ).

For some simple mathematical intuition into the nature of (2.21), observe that if $n=1$, and if we consider a constant concentration, zero yield stress and a concentric annulus, then (2.21) is simply:

$$
\frac{\rho}{H} \triangle \Psi_{t}=-3\left(\kappa+\mu_{\infty}\right) \triangle \Psi
$$

with suitable boundary conditions and initial condition. Evidently, it is possible to solve this simple linear problem, even analytically. The difficulties with (2.21) therefore come from both the non-linearity of the operator and a potential free boundary (between static and mobile fluid).

Our analysis proceeds as follows. We start with some initial assumptions and preliminary results that establish the relevant functional space for $\Psi$. We then characterise $\mathbf{S}$ as the subdifferential of a given functional and give a more rigorous definition of the evolution problem for $\Psi$. We demonstrate that there exists a unique solution to the free boundary problem for $\Psi$. This is followed by a number of continuity results, with respect to the problem data, and finally by some qualitative results on decay to the steady state. 


\subsection{Preliminary assumptions and results}

The following physically motivated assumptions are adopted throughout.

A1 The concentration $\bar{c}(\phi, \xi) \in H^{1}(\Omega)$, and is bounded by 0 and 1 . The physical properties of the fluid are all smooth functions of $\bar{c} ; \mu_{\infty}, \kappa, \rho$ and $n$ are strictly positive, $\tau_{Y}$ is semi-positive. All are bounded above and in particular $n \leqslant 1$.

A2 The flow rate through the annulus, $Q(t)$, and the various pressure gradients in the annulus are bounded. With A1 above, this implies that $\left(\mathbf{S}+\rho \nabla \Psi_{t}\right) \in\left[L^{\infty}(\Omega)\right]^{2}$, since we have that

$$
\mathbf{S}+\frac{\rho}{H} \nabla \Psi_{t}=\left(-\frac{\partial p}{\partial \xi}-\frac{\rho \cos \beta}{S t^{*}}, \frac{\partial p}{\partial \phi}-\frac{\rho \sin \beta \sin \pi \phi}{S t^{*}}\right) .
$$

A3 The annulus eccentricity $e$ satisfies $0 \leqslant e<1$, which implies that $1+e \geqslant H(\phi) \geqslant$ $1-e>0$.

A4 As $\xi \rightarrow 0$ and $\xi \rightarrow Z$, the physical properties of the fluid, $\tau_{Y}, \mu_{\infty}, \kappa, \rho$ and $n$ all approach constant values.

\subsubsection{Properties of $\chi(|\nabla \Psi|)$}

Proposition 1 For $|\nabla \Psi|>0$ we have that $\chi(|\nabla \Psi|)$ is $C^{\infty}$, strictly positive and strictly monotone; $\chi(|\nabla \Psi|) \rightarrow 0$ as $|\nabla \Psi| \rightarrow 0$.

Proposition 2 The function $\chi(|\nabla \Psi|)$ is bounded below by $\chi_{N}(|\nabla \Psi|), \quad \chi_{B}(|\nabla \Psi|)$ and $\chi_{H B}(|\nabla \Psi|)$, defined implicitly for $|\nabla \Psi| \geqslant 0$ as follows:

$$
\begin{aligned}
& |\nabla \Psi|=\frac{H^{3} \chi_{N}}{3 \mu_{\infty}}, \\
& |\nabla \Psi|=\frac{H^{3} \chi_{B}^{2}}{3 \mu_{\infty}} \frac{\left(\chi_{B}+1.5 \tau_{Y} / H\right)}{\left(\chi_{B}+\tau_{Y} / H\right)^{2}}, \\
& |\nabla \Psi|=\frac{H^{m+2}}{(m+2) \kappa} \frac{\chi_{H B}^{m+1}}{\left(\chi_{H B}+\tau_{Y} / H\right)^{2}}\left(\chi_{H B}+\frac{m+2}{m+1} \frac{\tau_{Y}}{H}\right), \quad m=1 / n .
\end{aligned}
$$

\section{Remarks:}

(1) See [13] for complete proofs of the above, which are essentially algebraic. In outline, proposition 1 follows directly from the properties of $A(|\nabla \Psi|)$, as discussed in Section 2.3. Proposition 2 follows from (2.15) and (2.16). For the bound with $\chi_{N}$, we bound $\tau(\dot{\gamma})$ below for $\tau \geqslant \tau_{Y}$, by neglecting the yield stress terms as well as the term $\kappa \dot{\gamma}^{n}$. For $\chi_{B}$, we bound $\tau(\dot{\gamma})$ below by neglecting the term $\kappa \dot{\gamma}^{n}$, and for $\chi_{H B}$, we bound $\tau(\dot{\gamma})$ below by neglecting the term $\mu_{\infty} \dot{\gamma}$. We insert the resulting bounds into (2.16) and perform the integrations; (3.2), (3.3) and (3.4) follow directly.

(2) The asymptotic behaviour as $|\nabla \Psi| \rightarrow \infty$ is of most interest. For $\chi_{H B}$, following [15], we have $\chi_{H B} \sim C_{H B}|\nabla \Psi|^{n}$, whereas evidently $\chi_{N} \sim C_{N}|\nabla \Psi|$ and $\chi_{B} \sim C_{B}|\nabla \Psi|$, where $C_{H B}=\left(H^{m+2} /(\kappa(m+2))\right)^{(-n)}$ and $C_{N}=C_{B}=3 \mu_{\infty} / H^{3}$. 
(3) It is also relatively straightforward to provide an upper bound for $\chi$. For example, writing

$$
\tau(\dot{\gamma}) \leqslant 2 \max \left\{\mu_{\infty} \dot{\gamma}, \kappa \dot{\gamma}^{n}\right\}+\tau_{Y},
$$

implies that $\dot{\gamma}(\tau) \geqslant \dot{\gamma}_{m}(\tau)$, where

$$
\dot{\gamma}_{m}(\tau)=\min \left\{\frac{\tau-\tau_{Y}}{2 \mu_{\infty}},\left[\frac{\tau-\tau_{Y}}{2 \kappa}\right]^{1 / n}\right\} .
$$

Therefore, defining $\chi_{M}$ implicitly via

$$
|\nabla \Psi|=\frac{1}{\left[\chi_{M}+\tau_{Y} / H\right]^{2}} \int_{\tau_{Y}}^{\chi_{M} H+\tau_{Y}} \tau \dot{\gamma}_{m}(\tau) \mathrm{d} \tau,
$$

leads to $\chi(|\nabla \Psi|) \leqslant \chi_{M}(|\nabla \Psi|)$. Furthermore, we can see that at large $\tau$,

$$
\dot{\gamma}_{m}(\tau)=\frac{\tau-\tau_{Y}}{2 \mu_{\infty}}
$$

so that

$$
|\nabla \Psi| \sim \frac{H^{3} \chi_{M}^{2}}{6 \mu_{\infty}} \frac{\left(\chi_{M}+1.5 \tau_{Y} / H\right)}{\left(\chi_{M}+\tau_{Y} / H\right)^{2}} \Rightarrow \chi_{M} \sim \frac{6 \mu_{\infty}|\nabla \Psi|}{H^{3}} .
$$

With the lower bounds in the above proposition, this demonstrates that $\chi(|\nabla \Psi|)$ is linear in the limit $|\nabla \Psi| \rightarrow \infty$.

(4) If we consider also the limit $|\nabla \Psi| \rightarrow 0$, when $\chi(|\nabla \Psi|) \rightarrow 0$, from the lower bound $\chi_{H B}(|\nabla \Psi|)$ and from the results in [15], we can establish that $\chi(|\nabla \Psi|) \gtrsim C|\nabla \Psi|^{1 /(1+m)}$ for positive $C$ in this limit. This may be used to establish the ellipticity of the steady problem.

\subsubsection{Behaviour of $\|\Psi\|$}

Lemma 1 Provided that assumptions A1, A2 and A3 hold, then the solution $\Psi$ of (2.21), satisfying boundary conditions (2.25), (2.26), (2.30) and (2.31) lies in the space

$$
\Psi \in L^{\infty}\left([0, \infty], H^{1}(\Omega)\right) .
$$

Proof First, let us comment that the boundary conditions (2.25), (2.26), (2.30) and (2.31) are compatible and give a boundary stream function that is continuous at the corners of the rectangular domain (and $C^{1}$ elsewhere).

Multiplying (2.21) by $\Psi$ and integrating over $\Omega$, using the divergence theorem:

$$
\int_{\Omega} \frac{\rho}{H} \nabla \Psi \cdot \nabla \Psi_{t} \mathrm{~d} \Omega=\int_{\partial \Omega} \Psi\left[\frac{\rho}{H} \nabla \Psi_{t}+\mathbf{S}\right] \cdot \boldsymbol{v} \mathrm{d} s-\int_{\Omega} \nabla \Psi \cdot \mathbf{S}-\Psi \nabla \cdot \mathbf{f} \mathrm{d} \Omega,
$$

where $v$ denotes the outward normal to $\Omega$. Using (3.1), we have

$$
\begin{aligned}
\int_{\partial \Omega} \Psi\left[\frac{\rho}{H} \nabla \Psi_{t}+\mathbf{S}\right] \cdot \boldsymbol{v} \mathrm{d} s & =\int_{\partial \Omega} \Psi\left(-\frac{\partial p}{\partial \xi}+g_{\xi}, \frac{\partial p}{\partial \phi}-g_{\phi}\right) \cdot \boldsymbol{v} \mathrm{d} s \\
& \leqslant\left\|\nabla p-\left(g_{\phi}, g_{\xi}\right)\right\|_{L^{\infty}(\partial \Omega)} \int_{\partial \Omega}|\Psi| \mathrm{d} s \\
& \leqslant C_{0}\left\|\nabla p-\left(g_{\phi}, g_{\xi}\right)\right\|_{L^{\infty}(\partial \Omega)}\|\Psi\|_{H_{\mathrm{s}}^{1}} .
\end{aligned}
$$


The first inequality follows from assumptions A1 and A2, using the Hölder inequality. The last line follows from

$$
\begin{aligned}
\int_{-L}^{L}\left|\frac{\partial \Psi}{\partial \xi}\right| \mathrm{d} \phi & \geqslant\left|\Psi_{\text {in }}\right|-\left|\Psi_{\text {out }}\right| \\
\Rightarrow & \int_{\Omega}\left|\frac{\partial \Psi}{\partial \xi}\right| \mathrm{d} \Omega \geqslant \int_{\partial \Omega_{1}}\left|\Psi_{\text {in }}\right| \mathrm{d} \phi+\int_{\partial \Omega_{3}}\left|\Psi_{\text {out }}\right| \mathrm{d} \phi \\
& \int_{\Omega}\left|\frac{\partial \Psi}{\partial \phi}\right| \mathrm{d} \Omega \geqslant \int_{\partial \Omega_{2}}|\Psi(1, \xi, t)| \mathrm{d} \xi+\int_{\partial \Omega_{4}}|\Psi(0, \xi, t)| \mathrm{d} \xi \\
\Rightarrow & 2 \int_{\Omega}|\nabla \Psi| \mathrm{d} \Omega \geqslant \int_{\partial \Omega}|\Psi| \mathrm{d} s
\end{aligned}
$$

From Proposition 2 we have that

$$
\chi(|\nabla \Psi|) \geqslant \frac{3 \mu_{\infty}}{H^{3}}|\nabla \Psi|
$$

Combining all this

$$
\begin{aligned}
\frac{\mathrm{d}}{\mathrm{d} t} \int_{\Omega} \frac{\rho}{2 H}|\nabla \Psi|^{2} \mathrm{~d} \Omega \leqslant & C_{0}\left\|\nabla p-\left(g_{\phi}, g_{\xi}\right)\right\|_{L^{\infty}(\partial \Omega)}\|\Psi\|_{H_{\mathrm{s}}^{1}}+\|\nabla \cdot \mathbf{f}\|_{L^{2}}\|\Psi\|_{L^{2}} \\
& -3 \inf _{\Omega}\left\{\frac{\mu_{\infty}}{H^{3}}\right\}\|\Psi\|_{H_{\mathrm{s}}^{1}}^{2}-\inf _{\Omega}\left\{\frac{\tau_{Y}}{H}\right\}\|\Psi\|_{H_{\mathrm{s}}^{1}}
\end{aligned}
$$

where

$$
\|\Psi\|_{H_{\mathrm{s}}^{1}}=\left[\int_{\Omega}|\nabla \Psi|^{2} \mathrm{~d} \Omega\right]^{1 / 2} .
$$

Since $\Psi=0$ along $\phi=0$, the seminorm $\|\Psi\|_{H_{\mathrm{s}}^{1}}$ is equivalent to $\|\Psi\|_{H^{1}}$, and evidently $\|\Psi\|_{L^{2}} \leqslant\|\Psi\|_{H^{1}}$. Therefore, we can find constants $C_{1} \geqslant 0$ and $C_{2}>0$, for which

$$
\frac{\mathrm{d}}{\mathrm{d} t} \int_{\Omega} \frac{\rho}{2 H}|\nabla \Psi|^{2} \mathrm{~d} \Omega \leqslant C_{1}\|\Psi\|_{H^{1}}-C_{2}\|\Psi\|_{H^{1}}^{2} .
$$

Integrating (3.9) with respect to $t$, we can find $C_{3}>0$ for which

$$
\begin{aligned}
C_{3}\|\Psi\|_{H^{1}}^{2}(t) & \leqslant \inf _{\Omega}\left\{\frac{\rho}{2 H}\right\}\|\Psi\|_{H_{s}^{1}}^{2}(t) \\
& \leqslant\left.\int_{\Omega} \frac{\rho}{2 H}|\nabla \Psi|^{2} \mathrm{~d} \Omega\right|_{t=0}+\int_{0}^{t} C_{1}\|\Psi\|_{H^{1}}(s)-C_{2}\|\Psi\|_{H^{1}}^{2}(s) \mathrm{d} s .
\end{aligned}
$$

We see that the integrand becomes negative if

$$
\|\Psi\|_{H^{1}}(t) \geqslant \frac{C_{1}}{C_{2}}
$$

and consequently $\|\Psi\|_{H^{1}}(t)$ is bounded for all $t>0$. 
3.1.3 $I(u), \partial I_{\mathrm{v}}$ and the steady-state problem

Denote by $V$ the subspace of $H^{1}(\Omega)$ containing functions that satisfy boundary conditions (2.25), (2.26), (2.30) and (2.31). The space $V$ is non-empty since for example $\Psi^{*} \in V$, where $\Psi^{*}=\left(\Psi_{\text {in }}(\phi, t)\left[\rho_{\text {out }}-\rho(\bar{c})\right]+\Psi_{\text {out }}(\phi, t)\left[\rho(\bar{c})-\rho_{\text {in }}\right]\right) /\left(\rho_{\text {out }}-\rho_{\text {in }}\right)$, and $\rho_{\text {in }} \& \rho_{\text {out }}$ are the density of pure fluids 1 and 2 , at the inflow and outflow, respectively. Note that the boundary streamfunctions, $\Psi_{\text {in }} \& \Psi_{\text {out }}$ must satisfy $\Psi_{\text {in }}(0, t)=\Psi_{\text {out }}(0, t)=0$ and $\Psi_{\text {in }}(1, t)=\Psi_{\text {out }}(1, t)=Q(t)$.

We now denote by $V_{0}$ the subspace of $H^{1}(\Omega)$ containing functions that are zero at $\partial \Omega$, i.e. $V_{0}=H_{0}^{1}(\Omega)$. Note that $V$ is an affine space, i.e. for any $\Psi^{*} \in V: V=\Psi^{*}+V_{0}$. Except where stated below, we shall now regard $\Psi^{*}$ as fixed and in particular time-invariant, i.e. the flow rate is constant.

We denote the usual $L^{2}(\Omega)$ inner product by $\langle\cdot, \cdot\rangle_{\mathrm{v}}$. For $\Psi^{*} \in V$ and $u \in L^{2}(\Omega)$, consider the following functional, $I(u)$ :

$$
I[u]:=I_{1}[u]+I_{2}[u]: \quad u \in L^{2}(\Omega),
$$

where

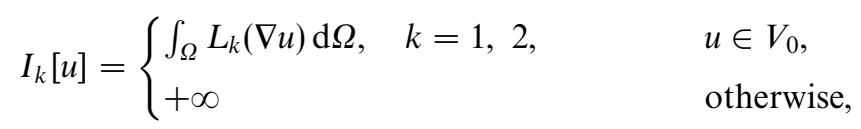

with

$$
\begin{aligned}
& L_{1}(\nabla u)=\frac{1}{2} \int_{0}^{\left|\nabla \Psi^{*}+\nabla u\right|^{2}} \frac{\chi\left(s^{1 / 2}\right)}{s^{1 / 2}} \mathrm{~d} s+\nabla\left(\Psi^{*}+u\right) \cdot \mathbf{f}, \\
& L_{2}(\nabla u)=\frac{\tau_{Y}}{H}\left|\nabla \Psi^{*}+\nabla u\right| .
\end{aligned}
$$

The sub-differential of $I$ is denoted $\partial I_{\mathrm{v}}$ and defined as follows:

$$
\partial I_{\mathrm{v}}[u]:=\left\{v \in L^{2}(\Omega): I[w] \geqslant I[u]+\langle v, w-u\rangle_{\mathrm{v}} \forall w \in V_{0}\right\} .
$$

We now give some results concerning the functional $I(u)$ and its sub-differentials. The following two results are proven in [13].

Proposition 3 The functional $I(u)$ is strictly convex on $L^{2}(\Omega)$, proper and lower semicontinuous on $L^{2}(\Omega) ; \partial I_{\mathrm{v}}[u]$ is monotone.

Proof Note that $L(\nabla u)=L_{1}(\nabla u)+L_{2}(\nabla u)$ is convex. Therefore, $L(\nabla u)$ is weakly lower semi-continuous. The lower semi-continuity follows from the weak lower semi-continuity. Lastly, since $I[u]$ is convex, proper and lower semi-continuous, $\partial I[u]$ is monotone.

Proposition 4 For $u \in V_{0}$, let $I[u]=I_{1}[u]+I_{2}[u]$, with $I_{1}[u]$ Gâteaux-differentiable and $\partial I_{1, V}[u]$ single valued. Then if $v_{1} \in \partial I_{1, V}[u]$

$$
v=v_{1}+v_{2} \in \partial\left(I_{1, V}[u]+I_{2, V}[u]\right) \Leftrightarrow v_{2} \in \partial I_{2, V}[u] .
$$


Proof $\Rightarrow$ : Because $v_{1} \in \partial I_{1}[u]$ and $I_{1}[u]$ is Gâteaux-differentiable, we have

$$
I_{1}[u]-I_{1}[w] \geqslant\left(w-u,-v_{1}\right), \quad \forall w \in V_{I, 0},
$$

i.e. because $\partial I_{1}[u]$ is single valued. We know that $v_{1}+v_{2} \in \partial\left(I_{1}[u]+I_{2}[u]\right)$, thus

$$
I_{1}[w]-I_{1}[u]+I_{2}[w]-I_{2}[u] \geqslant\left(w-u, v_{1}+v_{2}\right) \forall w \in V_{I, 0} .
$$

Adding (3.15) and (3.16), we get

$$
I_{2}[w]-I_{2}[u] \geqslant\left(w-u, v_{2}\right) \forall w \in V_{I, 0} .
$$

Therefore, $v_{2} \in \partial I_{2}[u]$.

$\Leftarrow$ : By assumption $\partial I_{1}[u]$ is single valued and $v_{1} \in \partial I_{1}[u]$. Therefore, because $v_{2} \in \partial I_{2}[u]$, (3.16) and (3.17) are satisfied. Hence $v_{1}+v_{2} \in \partial\left(I_{1}[u]+I_{2}[u]\right)$.

In Proposition 4, $\partial I_{k, V}[u]$ denotes the sub-differential of $I_{k}[u]$, with respect to the inner product $\langle\cdot, \cdot\rangle_{\mathrm{v}}$, defined as per (3.14). We may now characterise the sub-differential $\partial I_{V}[u]$. The first functional $I_{1}[u]$ is Gâteaux-differentiable, consequently $\partial I_{1, V}[u]=I_{1, V}^{\prime}[u]$, and we find that

$$
I_{1}[w] \geqslant I_{1}[u]-\int_{\Omega}(w-u) \nabla \cdot\left(\frac{\chi\left(\left|\nabla \Psi^{*}+\nabla u\right|\right)}{\left|\nabla \Psi^{*}+\nabla u\right|}\left(\nabla \Psi^{*}+\nabla u\right)+\mathbf{f}\right) \mathrm{d} \Omega
$$

for $u \in V_{0}, \forall w \in V_{0}$. For the second functional (see [1]), if $u \in V_{0}$, then $\partial I_{2, V}[u]$ is characterized by

$$
-\nabla \cdot\left(\frac{\tau_{Y}}{H} \frac{\left(\nabla \Psi^{*}+\nabla u\right)}{\left|\nabla \Psi^{*}+\nabla u\right|}\right)
$$

which is set-valued for $\left|\nabla \Psi^{*}+\nabla u\right|=0$. Thus

$$
I_{2}[w] \geqslant I_{2}[u]-\int_{\Omega}(w-u) \nabla \cdot\left(\frac{\tau_{Y}}{H} \frac{\left(\nabla \Psi^{*}+\nabla u\right)}{\left|\nabla \Psi^{*}+\nabla u\right|}\right) \mathrm{d} \Omega .
$$

We now combine these two terms, using Proposition 4, to give

$$
I[w] \geqslant I[u]-\int_{\Omega}(w-u) \nabla \cdot(\mathbf{S}[u]+\mathbf{f}) \mathrm{d} \Omega, \quad u \in V_{I, 0}, \forall w \in V_{I, 0}
$$

i.e. $v \in \partial I_{V}[u] \Rightarrow v \in-\nabla \cdot(\mathbf{S}[u]+\mathbf{f})$.

\subsection{Steady-state problem}

We may now consider the steady problem for $\Psi$, which may be written as

$$
\nabla \cdot(\mathbf{S}+\mathbf{f}) \ni 0
$$

with boundary conditions (2.25), (2.26), (2.30) and (2.31). 
Theorem 1 [Steady-state problem] There exists a unique solution $\Psi_{\mathrm{s}} \in V_{I}$ to (3.21), where $\Psi=\Psi^{*}+u_{\mathrm{s}}$, and $u_{\mathrm{s}}$ is the minimiser of

$$
\inf _{v \in V_{0}} I[v]
$$

Proof This is essentially the same result as in [15] except that, due to adoption of (2.6), we are now in the Hilbert space setting.

\subsection{Solution to the transient problem}

We now consider the existence of a solution to the transient problem. For simplicity we assume a constant flow rate and again consider $\Psi^{*}$ to be fixed. We shall need to consider the elliptic problem

$$
E[z]=-v: \quad E[z]=\nabla \cdot\left[\frac{\rho}{H} \nabla z\right], \quad \text { in } \Omega, \quad z=0 \text { on } \partial \Omega .
$$

Since assumptions A1-A3 are satisfied, $0<\frac{\rho_{\min }}{1+e} \leqslant \frac{\rho(\bar{c})}{H} \leqslant \frac{\rho_{\max }}{1-e}$. Therefore, $E$ is a strictly elliptic operator with bounded coefficients, and for $v \in H^{-1}(\Omega)$, this problem has a unique solution $z \in H_{0}^{1}(\Omega)$ (see [10], Theorem 8.3 and Corollary 8.7, p. 171). Associated with this elliptic problem, we denote by $U_{0}=H_{0}^{1}(\Omega)$, the Hilbert space equipped with the inner product $\langle\cdot, \cdot\rangle_{U}$ :

$$
\langle v, w\rangle_{U}=\int_{\Omega} \frac{\rho}{H} \nabla v \cdot \nabla w \mathrm{~d} \Omega, \quad \forall v, w \in U_{0} .
$$

We shall denote the seminorm associated with (3.24) by $\|\cdot\|_{U}$. Since $0<\rho_{\min } \leqslant \rho \leqslant \rho_{\max }$ and $1-e \leqslant H \leqslant 1+e$, the associated seminorm is equivalent to $\|\cdot\|_{H^{1}}$ on $U_{0}$, and in fact we can show that $U_{0}=H_{0}^{1}(\Omega)$. We define $E^{-1}: H^{-1}(\Omega) \mapsto U_{0}$ as the solution to (3.23), i.e. $E^{-1}[v]=z$. Note that for any $\Psi^{*} \in V$, since $V_{0}=H_{0}^{1}(\Omega)=U_{0}$, we have that $V=\Psi^{*}+U_{0}$.

Evidently, $I[u]$ is defined for $u \in U_{0}$. The sub-differential of $I[u]$ with respect to $U_{0}$ is defined as

$$
\partial I_{U}[u]:=\left\{z \in U_{0}: I[w] \geqslant I[u]+<z, w-u>_{U}, \quad \forall w \in U_{0}, u \in U_{0}\right\} .
$$

Suppose that $z \in \partial I_{U}[u]$. It follows that for $u \in U_{0}$ and $\forall w \in U_{0}$, we have that

$$
\begin{aligned}
& I[w] \geqslant I[u]+<z, w-u>_{U} \\
& I[w] \geqslant I[u]-<E[z], w-u>_{\mathrm{v}},
\end{aligned}
$$

and therefore, $-E[z] \in \partial I_{V}[u]$. According to our characterisation of $\partial I_{V}[u]$, this implies that

$$
E[z]=\nabla \cdot(\mathbf{S}[u]+\mathbf{f}) .
$$

Conversely, suppose that $v \in \partial I_{V}[u]$, which we have characterised by $-\nabla \cdot(\mathbf{S}[u]+\mathbf{f})$. Then, we have that $v \in-\nabla \cdot(\mathbf{S}[u]+\mathbf{f}) \subset L^{2}(\Omega)$, which is embedded in $H^{-1}(\Omega)$, so that $E^{-1}[v]$ is 
well-defined. From (3.20) we can write

$$
\begin{aligned}
-\int_{\Omega}(w-u) \nabla \cdot(\mathbf{S}[u]+\mathbf{f}) \mathrm{d} \Omega & =-\int_{\Omega}(w-u) E\left[E^{-1} \nabla \cdot(\mathbf{S}[u]+\mathbf{f})\right] \mathrm{d} \Omega \\
& =-\int_{\Omega}(w-u) \nabla \cdot\left(\frac{\rho}{H} \nabla\left[E^{-1} \nabla \cdot(\mathbf{S}[u]+\mathbf{f})\right]\right) \mathrm{d} \Omega \\
& =\int_{\Omega} \frac{\rho}{H} \nabla(w-u) \cdot \nabla\left[E^{-1} \nabla \cdot(\mathbf{S}[u]+\mathbf{f})\right] \mathrm{d} \Omega
\end{aligned}
$$

Therefore, if $v \in \partial I_{V}[u] \Rightarrow E^{-1}[-v] \in \partial I_{U}[u]$. Similarly, we may show that if $u \in D\left(\partial I_{V}\right)$ then $u \in D\left(\partial I_{U}\right)$.

We are now in a position to demonstrate existence of a solution. Consider the differential equation:

$$
\left\{\begin{array}{l}
u^{\prime}(t)+A[u(t)] \ni 0 \quad t \geqslant 0, \\
u(0)=u_{0}
\end{array}\right.
$$

where $u^{\prime}(t)$ denotes the time derivative of $u, u_{0} \in H$ is given and $H$ is a Hilbert space; $A=\partial I$ is the subgradient of $I$, which may be non-linear and perhaps multi-valued. Our result follows from the application of the following theorem.

Theorem 2 For each $u_{0} \in D(\partial I)$ there exists a unique function

$$
u \in C([0, \infty) ; H) \text { with } u^{\prime} \in L^{\infty}(0, \infty ; H)
$$

such that,

(1) $u(0)=u_{0}$,

(2) $u(t) \in D(\partial I)$ for each $t>0$, and

(3) $-u^{\prime}(t) \in \partial I$ for a.e. $t \geqslant 0$.

Proof See [7] (p. 529-533).

We now apply this theorem to the differential equation

$$
\left\{\begin{array}{l}
u^{\prime}(t)+\partial I_{U}[u(t)] \ni 0 \quad t \geqslant 0 \\
u(0)=u_{0}
\end{array}\right.
$$

It follows that there exists a unique function

$$
u \in C\left([0, \infty) ; U_{0}\right) \text { with } u^{\prime} \in L^{\infty}\left(0, \infty ; U_{0}\right)
$$

such that

(1) $u(0)=u_{0}$,

(2) $u(t) \in D\left(\partial I_{U}\right)$ for each $t>0$, and

(3) $-u^{\prime}(t) \in \partial I_{U}$ for a.e. $t \geqslant 0$. 
We have seen that if $z \in \partial I_{U}$, then $-E[z] \in \partial I_{\mathrm{v}}$. It follows that we have $u$ satisfying:

$$
E\left[u^{\prime}(t)\right] \in \partial I_{\mathrm{v}} \text { for a.e. } t \geqslant 0 \Longrightarrow E\left[u^{\prime}(t)\right]+\nabla \cdot(\mathbf{S}[u]+\mathbf{f}) \ni 0 .
$$

The latter is our evolution equation for $\Psi$ (note that $E\left[u^{\prime}(t)\right]=E\left[\Psi^{\prime}(t)\right]$ ). Therefore equation (2.21), in the above form, has a unique solution $u \in C\left([0, \infty) ; U_{0}\right)$ and (see earlier comments), $U_{0}$ is $H_{0}^{1}(\Omega)$.

\subsection{Continuity with respect to physical parameters}

It is possible to prove the continuity of the solution with respect to many of the physical parameters. The proofs of the following results are lengthy and mostly technical; they may be found in [13]. In what follows, we assume that $\Psi_{1}, \Psi_{2} \in H^{1}(\Omega)$ are two solutions that correspond to variation of a single physical properties, as indicated, e.g. varying density: $\rho_{1}(\phi, \xi) \mapsto \Psi_{1}$ and $\rho_{2}(\phi, \xi) \mapsto \Psi_{2}$, with all other physical properties remaining identical. The solutions $\Psi_{1}$ and $\Psi_{2}$ may be shown to satisfy the following continuity bounds.

Proposition 5 [Steady problem] For two steady solutions for which one of the physical properties varies, we may find strictly positive constants $C$, independent of the solutions, such that

$$
\begin{aligned}
\left\|\rho_{1}-\rho_{2}\right\|_{L^{\infty}} & \geqslant C\left\|\nabla\left(\Psi_{1}-\Psi_{2}\right)\right\|_{L^{2}}^{2}, \\
\left\|\tau_{Y, 1}-\tau_{Y, 2}\right\|_{L^{\infty}} & \geqslant C\left\|\nabla\left(\Psi_{1}-\Psi_{2}\right)\right\|_{L^{2}}^{2}, \\
\left\|\kappa_{1}-\kappa_{2}\right\|_{L^{\infty}} & \geqslant C\left\|\nabla\left(\Psi_{1}-\Psi_{2}\right)\right\|_{L^{2}}^{2}, \\
\left\|\mu_{\infty, 1}-\mu_{\infty, 2}\right\|_{L^{\infty}} & \geqslant C\left\|\nabla\left(\Psi_{1}-\Psi_{2}\right)\right\|_{L^{2}}^{2} .
\end{aligned}
$$

Proposition 6 [Transient problem] For two transient solutions for which one of the physical properties varies, we may find strictly positive constants $C$, independent of the solutions, such that for $t<T$ :

$$
\begin{array}{r}
\left\|\rho_{1}-\rho_{2}\right\|_{L^{\infty}}+\left\|\nabla\left(\Psi_{1}-\Psi_{2}\right)\right\|_{L^{2}}^{2}(0) \geqslant C\left\|\nabla\left(\Psi_{1}-\Psi_{2}\right)\right\|_{L^{2}}^{2}(t), \\
\left\|\tau_{Y, 1}-\tau_{Y, 2}\right\|_{L^{\infty}}+\left\|\nabla\left(\Psi_{1}-\Psi_{2}\right)\right\|_{L^{2}}^{2}(0) \geqslant C\left\|\nabla\left(\Psi_{1}-\Psi_{2}\right)\right\|_{L^{2}}^{2}(t), \\
\left\|\kappa_{1}-\kappa_{2}\right\|_{L^{\infty}}+\left\|\nabla\left(\Psi_{1}-\Psi_{2}\right)\right\|_{L^{2}}^{2}(0) \geqslant C\left\|\nabla\left(\Psi_{1}-\Psi_{2}\right)\right\|_{L^{2}}^{2}(t), \\
\left\|\mu_{\infty, 1}-\mu_{\infty, 2}\right\|_{L^{\infty}}+\left\|\nabla\left(\Psi_{1}-\Psi_{2}\right)\right\|_{L^{2}}^{2}(0) \geqslant C\left\|\nabla\left(\Psi_{1}-\Psi_{2}\right)\right\|_{L^{2}}^{2}(t) .
\end{array}
$$

We note that we have not been able to establish continuity results with respect to variations in the power law index between two solutions.

\subsection{Decay to the steady state}

If the concentration field and flow rate remain steady, we might expect that the solution of the transient model will decay to that of the steady model, as $t \rightarrow \infty$. This is indeed the case, as we see below. 
For the rest of this section, we assume that $\Psi^{*}=\Psi_{\mathrm{s}}$ (the steady solution). Multiplying (1.1) by $\left(\Psi-\Psi_{\mathrm{s}}\right)$ and integrating by parts gives us

$$
\int_{\Omega}\left(\frac{\chi\left(\left|\nabla \Psi_{\mathrm{s}}\right|\right)}{\left|\nabla \Psi_{\mathrm{s}}\right|} \nabla \Psi_{\mathrm{s}}\right) \cdot \nabla\left(\Psi-\Psi_{\mathrm{s}}\right)+\frac{\tau_{Y}}{H}\left(|\nabla \Psi|-\left|\nabla \Psi_{\mathrm{s}}\right|\right)+\mathbf{f} \cdot \nabla\left(\Psi-\Psi_{\mathrm{s}}\right) \mathrm{d} \Omega \geqslant 0 .
$$

Note that $\left(\Psi-\Psi_{\mathrm{s}}\right)$ vanishes on the boundary. We also multiply $(2.21)$ by $\left(\Psi_{\mathrm{s}}-\Psi\right)$ and integrate by parts:

$$
\begin{aligned}
\int_{\Omega} \frac{\rho}{H} \nabla \Psi_{t} \cdot \nabla\left(\Psi_{\mathrm{s}}-\Psi\right) \mathrm{d} \Omega & +\int_{\Omega}\left(\frac{\chi(|\nabla \Psi|)}{|\nabla \Psi|} \nabla \Psi\right) \cdot \nabla\left(\Psi_{\mathrm{s}}-\Psi\right) \mathrm{d} \Omega \\
& +\int_{\Omega} \frac{\tau_{Y}}{H}\left(\left|\nabla \Psi_{\mathrm{s}}\right|-|\nabla \Psi|\right)+\mathbf{f} \cdot \nabla\left(\Psi_{\mathrm{s}}-\Psi\right) \mathrm{d} \Omega \geqslant 0
\end{aligned}
$$

In [13] (see proposition 10, p. 65), it is shown that

$$
\int_{\Omega}\left(\frac{\chi\left(\left|\nabla \Psi_{\mathrm{s}}\right|\right)}{\left|\nabla \Psi_{\mathrm{s}}\right|} \nabla \Psi_{\mathrm{s}}-\frac{\chi(|\nabla \Psi|)}{|\nabla \Psi|} \nabla \Psi\right) \cdot \nabla\left(\Psi_{\mathrm{s}}-\Psi\right) \mathrm{d} \Omega \leqslant-C\left\|\nabla\left(\Psi-\Psi_{\mathrm{s}}\right)\right\|_{L^{2}}^{2},
$$

for $C>0$ and independent of the solutions. Summing (3.28) and (3.29), we have

$$
\int_{\Omega} \frac{\rho}{H} \nabla \Psi_{t} \cdot \nabla\left(\Psi_{\mathrm{s}}-\Psi\right) \mathrm{d} \Omega-C\left\|\nabla\left(\Psi-\Psi_{\mathrm{s}}\right)\right\|_{L^{2}}^{2} \geqslant 0
$$

Because $\frac{\partial \Psi_{s}}{\partial t}=0,(3.31)$ states that

$$
\frac{1}{2} \frac{\mathrm{d}}{\mathrm{d} t} \int_{\Omega} \frac{\rho}{H}\left|\nabla\left(\Psi_{\mathrm{s}}-\Psi\right)\right|^{2} \mathrm{~d} \Omega+C\left\|\nabla\left(\Psi-\Psi_{\mathrm{s}}\right)\right\|_{L^{2}}^{2} \leqslant 0 .
$$

Since $\rho$ is positive and bounded, we have via Gronwall's lemma:

$$
\left\|\Psi-\Psi_{\mathrm{s}}\right\|_{U}(t) \leqslant\left\|\Psi-\Psi_{\mathrm{s}}\right\|_{U}(0) e^{-K t}
$$

which translates to

$$
\left\|\nabla\left(\Psi-\Psi_{\mathrm{s}}\right)\right\|_{L^{2}}(t) \leqslant R_{\rho}\left\|\nabla\left(\Psi-\Psi_{\mathrm{s}}\right)\right\|_{L^{2}}(0) e^{-K t}
$$

where

$$
R_{\rho}=\frac{\rho_{\max }(1+e)}{\rho_{\min }(1-e)} \quad \text { and } \quad K=2 C .
$$

Thus, we have exponential decay to the steady state solution, as expected.

\subsubsection{Finite time decay}

A special case of the above occurs when the steady-state solution is $\Psi_{\mathrm{s}}=0$. It is obvious that a necessary condition for this to happen is that $Q=0$, in which case all the boundary values are zero. To find a sufficient condition, we multiply (3.21) by $\Psi_{\mathrm{s}}$ and integrate by 
parts to give

$$
\begin{aligned}
\int_{\Omega} \chi\left(\left|\nabla \Psi_{\mathrm{s}}\right|\right)\left|\nabla \Psi_{\mathrm{s}}\right| \mathrm{d} \Omega & =-\int_{\Omega} \frac{\tau_{Y}}{H}\left|\nabla \Psi_{\mathrm{s}}\right| \mathrm{d} \Omega+\int_{\Omega} \Psi_{\mathrm{s}} \nabla \cdot \mathbf{f} \mathrm{d} \Omega \\
& \leqslant-\left(\frac{\tau_{Y, \text { min }}}{1+e}-\frac{\|\nabla \cdot \mathbf{f}\|_{L^{\infty}(\Omega)}}{2}\right) \int_{\Omega}\left|\nabla \Psi_{\mathrm{s}}\right| \mathrm{d} \Omega .
\end{aligned}
$$

Therefore, for $Q=0$ and

$$
\frac{\tau_{Y, \min }}{1+e} \geqslant \frac{\|\nabla \cdot \mathbf{f}\|_{L^{\infty}(\Omega)}}{2}
$$

we have that $\Psi_{\mathrm{s}}=0$.

Let us assume that (3.35) holds. Considering now our decay results above for the transient problem, we know that $\Psi \rightarrow 0$ at least exponentially. From the variational form of the transient problem, we have

$$
\int_{\Omega} \frac{\rho}{H} \nabla \Psi_{t} \cdot \nabla \Psi \mathrm{d} \Omega=-\int_{\Omega} \chi(|\nabla \Psi|)|\nabla \Psi|+\frac{\tau_{Y}}{H}|\nabla \Psi| \mathrm{d} \Omega+\int_{\Omega} \Psi \nabla \cdot \mathbf{f} \mathrm{d} \Omega .
$$

Using proposition 10 in [13] we have

$$
\begin{aligned}
\frac{1}{2} \frac{d}{d t}\|\Psi\|_{U}^{2} & \leqslant-C\|\Psi\|_{\dot{H}^{1}(\Omega)}^{2}-\left(\frac{\tau_{Y, \min }}{1+e}-\frac{\|\nabla \cdot \mathbf{f}\|_{L^{\infty}(\Omega)}}{2}\right) \int_{\Omega}|\nabla \Psi| \mathrm{d} \Omega \\
& \leqslant-C C_{\Omega}\|\Psi\|_{L^{2}(\Omega)}^{2}-B_{\Omega}\left(\frac{\tau_{Y, \min }}{1+e}-\frac{\|\nabla \cdot \mathbf{f}\|_{L^{\infty}(\Omega)}}{2}\right)\|\Psi\|_{L^{2}(\Omega)},
\end{aligned}
$$

where $C_{\Omega}$ is the Poincare constant for $\Omega$ and $B_{\Omega}$ is defined by

$$
B_{\Omega}=\inf _{v \in U, v \neq 0} \frac{\int_{\Omega}|\nabla v| \mathrm{d} \Omega}{\|v\|_{L^{2}(\Omega)}} .
$$

We may calculate that (for $Z>1$, as is usual), $C_{\Omega}=\pi^{2}$ and $B_{\Omega}=2$. Integrating with respect to $t$ gives us

$$
\begin{aligned}
\frac{\rho_{\min } \pi^{2}}{2(1+e)}\|\Psi\|_{L^{2}(\Omega)}^{2}(t) \leqslant & \frac{1}{2}\|\Psi\|_{U}^{2}(t) \\
\leqslant & \frac{1}{2}\|\Psi\|_{U}^{2}(0)-\pi^{2} C \int_{0}^{t}\|\Psi\|_{L^{2}(\Omega)}^{2}(s) \mathrm{d} s \\
& -2\left[\frac{\tau_{Y, \min }}{1+e}-\frac{\|\nabla \cdot \mathbf{f}\|_{L^{\infty}(\Omega)}}{2}\right] \int_{0}^{t}\|\Psi\|_{L^{2}(\Omega)}^{2}(s) \mathrm{d} s .
\end{aligned}
$$

If we take equality in the above, we have $z(t) \geqslant 0$ satisfying

$$
z^{2}(t)=z_{0}^{2}+2 \int_{0}^{t} \alpha_{1} z^{2}(s)+\alpha_{2} z(s) \mathrm{d} s
$$

with

$$
z_{0}=\frac{\|\Psi\|_{U}(0)(1+e)}{\pi \sqrt{\rho_{\min }}}, \quad \alpha_{1}=\frac{C(1+e)}{\rho_{\min }}, \quad \alpha_{2}=\frac{2(1+e)}{\rho_{\min } \pi^{2}}\left[\frac{\tau_{Y, \min }}{1+e}-\frac{\|\nabla \cdot \mathbf{f}\|_{L^{\infty}(\Omega)}}{2}\right] .
$$


The solution of this integral equation is for $0 \leqslant t \leqslant t_{\mathrm{s}}$ :

$$
z(t)=\left(z_{0}+\frac{\alpha_{2}}{\alpha_{1}}\right) \mathrm{e}^{-\alpha_{1} t}-\frac{\alpha_{2}}{\alpha_{1}},
$$

and $z(t)=0$ for $t>t_{\mathrm{s}}$, where

$$
t_{\mathrm{s}}=\frac{1}{\alpha_{1}} \ln \left[1+\frac{z_{0} \alpha_{1}}{\alpha_{2}}\right] .
$$

Thus, we may expect $\|\Psi\|_{L^{2}(\Omega)}(t)=0$ for $t>t_{\mathrm{s}}$. This bound is primarily of use as a computational test problem. For example, if we have a Bingham fluid with uniform physical properties initially flowing in a concentric annulus, then we have that $C \geqslant \kappa$, $\nabla \cdot \mathbf{f}=0$, and we have

$$
t_{\mathrm{s}}=\frac{\rho}{\kappa} \ln \left[1+\frac{\pi \kappa\|\Psi\|_{U}(0)}{2 \tau_{Y}}\right] \sim \frac{\pi \rho\|\Psi\|_{U}(0)}{2 \tau_{Y}}, \text { as } \tau_{Y} \rightarrow \infty .
$$

\section{Computational results}

We now consider solution of the fully coupled transient model:

$$
\nabla \cdot\left[\frac{\rho}{H} \nabla \Psi_{t}\right]=-\nabla \cdot[\mathbf{S}+\mathbf{f}]
$$

where

$$
\begin{gathered}
\mathbf{S}=\left(\frac{\chi(|\nabla \Psi|)+\tau_{Y} / H}{|\nabla \Psi|}\right) \nabla \Psi \Leftrightarrow|\mathbf{S}|>\tau_{Y} / H, \\
|\nabla \Psi|=0 \Leftrightarrow|\mathbf{S}| \leqslant \tau_{Y} / H, \\
\Psi(0, \xi, t)=0, \\
\Psi(1, \xi, t)=Q(t), \\
\Psi(\phi, 0, t)=\Psi_{\text {in }}(\phi, t), \\
\Psi(\phi, Z, t)=\Psi_{\text {out }}(\phi, t), \\
\frac{1}{\epsilon} \frac{\partial}{\partial t}[H \bar{c}]+\frac{\partial}{\partial \phi}[H \bar{v} \bar{c}]+\frac{\partial}{\partial \xi}[H \bar{w} \bar{c}]=0 .
\end{gathered}
$$

The system (4.1)-(4.8) is supplemented with closure laws for the fluid properties. For simplicity, we follow [3] and assume linear interpolation between the constant values of the pure fluids.

For the case where the flow rate and concentration are constant, we have established in the previous section that this model is well-posed. We first compute some test problems, verifying the decay results of Section 3.5, and then look at the problem of removing a static narrow-side mud channel via flow rate pulsation; see Section 4.3.

\subsection{Computational method outline}

To solve the system (4.1)-(4.8) numerically, a hybrid method is used. We discretise $(\phi, \xi) \in[0,1] \times[0, Z]$, using a rectangular mesh. The concentration equation is discretised 
using a conservative finite volume approximation at the middle point of each element and solved using a Flux Corrected Transport scheme. The FCT scheme is used to minimize both numerical dispersion and diffusion, see [19]. Although more sophisticated schemes are available, the primary limitations in terms of accuracy and computational speed come from solving (4.1).

There are two key problems in solving (4.1). Firstly, how to handle the yielding behaviour, i.e. the free boundary between static and mobile fluids. Secondly, how to handle the time evolution. In its steady form, (4.1) resembles the Dirichlet problem for the flow of a visco-plastic fluid through a duct (with in this case, $\Psi$ representing the axial velocity). The key issue for such flows is whether or not it is necessary to accurately model the unyielded flow regions, or whether it is adequate to use a regularisation method. In the present case, unyielded flow typically corresponds to unyielded drilling mud, and hence this is of importance. Consequently, we use the augmented Lagrangian method of $[8,11]$ on each time step. The time derivative is handled fully implicitly, which is known to preserve some of the time decay properties of systems such as (4.1)-(4.7), e.g. we would expect finite-time decay of $\Psi \rightarrow 0$ in the event that we set $Q(t)=0$. The spatial discretisation is with bilinear basis functions on quadrilateral finite elements. The disadvantage of the augmented Lagrangian approach is the large number of iterations that are needed. Further details of the implementation may be found in [13].

\subsection{Test computations}

There are no known non-trivial analytical (or semi-analytical) solutions to the system (4.1)-(4.8). For the steady problem, it is possible to find analytical solutions for steadily advancing displacements in concentric annuli (travelling wave solutions) and also asymptotic approximations may be used to extend these solutions to slightly eccentric annuli, $e \ll 1$; see [15]. If the fluid properties are constant throughout the annulus, then there exists a uni-directional flow solution, in which the streamlines are all parallel to the $\xi$-axis. Such solutions can be computed via solution of a single monotone non-linear equation, i.e. these are semi-analytical solutions. It is such uni-directional flow solutions that are used to define the end conditions, $\Psi_{\text {in }}(\phi, t)$ and $\Psi_{\text {out }}(\phi, t)$ in (4.6) \& (4.7). These test solutions are however not ideally suited to the transient problem.

Instead, as a first test for the transient numerical solution, we use the decay results from Section 3.5. We freeze the concentration field and compute a steady solution, $\Psi_{\mathrm{s}}$, using the same spatial discretisation and augmented Lagrangian algorithm as for the transient problem. We then solve the transient problem (4.1)-(4.7) and show that $\Psi \rightarrow \Psi_{\mathrm{s}}$ as $t \rightarrow \infty$ in the $H^{1}$ norm.

The rheological and physical parameters for fluid 1 are $\tau_{Y, 1}=1, \kappa_{1}=1, \rho_{1}=1, m_{1}=1$, $\mu_{1, \infty}=0$; for fluid 2 the properties are $\tau_{Y, 2}=1, \kappa_{2}=1, \rho_{2}=1, m_{2}=2, \mu_{2, \infty}=0$. The annulus is vertical, $\beta=0$, and is eccentric, $e=0.4$. The domain is $(\phi, \xi) \in[0,1] \times[0,5]$ and we allow the steady concentration to vary from 0 to 1 between $\xi=2$ and $\xi=3$ (see Figure $3 \mathrm{a}$ ). The steady state solution, $\Psi_{\mathrm{s}}(\phi, \xi)$, is shown in Figure $3(\mathrm{~b})$, and shows a smooth variation between the two parallel stream function solutions at inflow and outflow.

For the transient problem we maintain a steady flow rate $Q(t)=1$ and set as initial condition:

$$
\Psi(\phi, \xi, 0)=\sin (\pi \phi) \sin (\xi \pi)
$$



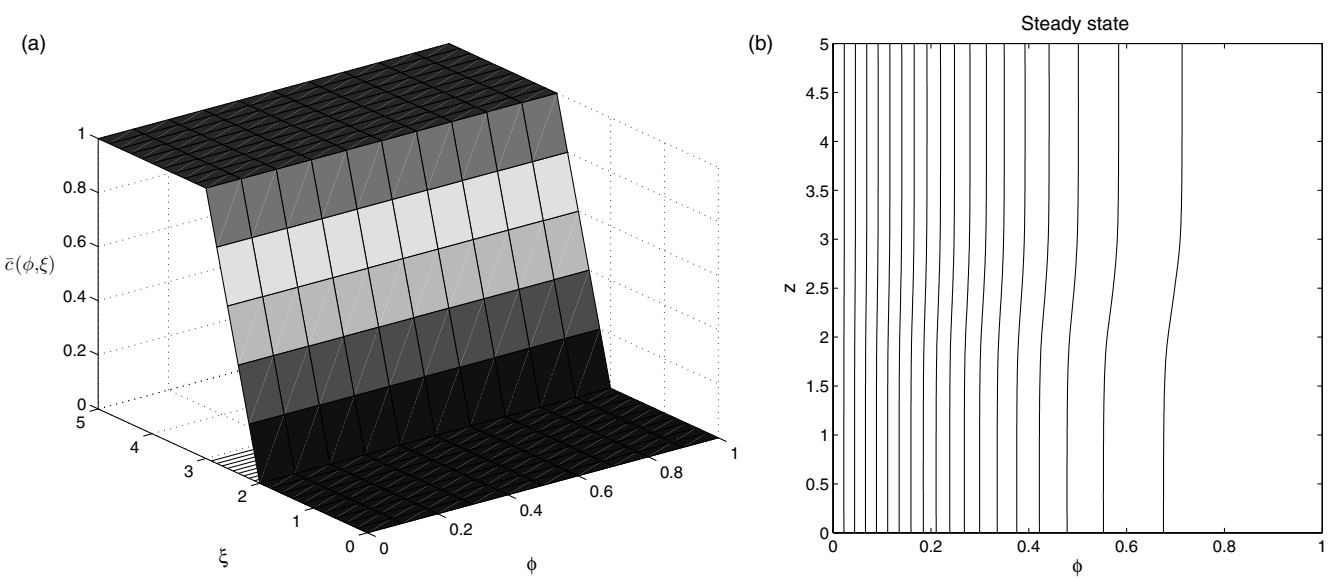

FIGURE 3. (a) the steady concentration field assumed for our test computation; (b) the steady-state solution $\Psi_{\mathrm{s}}(\phi, \xi)$, with contours at intervals $\Delta \Psi_{\mathrm{s}}=0.05$.

Figures 4(a)-(c) show decay of the streamfunction towards the steady state at different times. Decay of $\left\|\Psi-\Psi_{\mathrm{s}}\right\|_{L^{2}(\Omega)}$ as time evolves is shown in Figure 4(d), which we can see approaches an exponential decay, as predicted.

As a second test problem, we assume the same physical parameters and initial condition (4.9). We impose as flow rate, $Q(t)=0$, and we may deduce that $\Psi_{\mathrm{s}}=0$ for these physical parameters. We therefore expect to find finite time decay of the transient solution, following our analysis of the previous section. This is indeed found to be the case. Figure 5 shows the decay of $\|\Psi\|_{L^{2}(\Omega)}(t) \rightarrow 0$.

\subsection{Static mud channel removal}

For certain combinations of dimensionless parameters, it is possible for one of the fluids to be stationary on the narrow side of the annulus. Physically, this occurs wherever the wall shear stress is not large enough to overcome the yield stress of the fluid. From the industrial perspective this can be a serious problem if stationary drilling mud forms a long channel along the narrow side of the annulus. As the cement sets, water is sucked from the mud, which dries into a porous conduit along the length of the well. Such conduits connect reservoirs of different pressure, reducing productivity, and can also allow subsurface fluids to percolate towards the surface. We would like to know if such static channels can be removed or reduced by any means. In a companion paper we study whether interfacial instability might occur during the actual displacement, as the front elongates into a (pseudo-)parallel finger [14]. In this paper we investigate whether pulsation of the flow can reduce mud channel formation. As the problem is intractable analytically, we study it via numerical simulation.

For all the numerical results we show below, we set $\mu_{k, \infty}=0$. This is simpler (and quicker) to work with than $\mu_{\infty}>0$, as $\chi$ can be defined via an implicit algebraic expression; see [3]. The inclusion of $\mu_{\infty}>0$ affects the high-shear behaviour, whereas mud channel formation is related to the low-shear viscosity and the yield stress. Mathematically, when $\mu_{\infty}=0$ we 

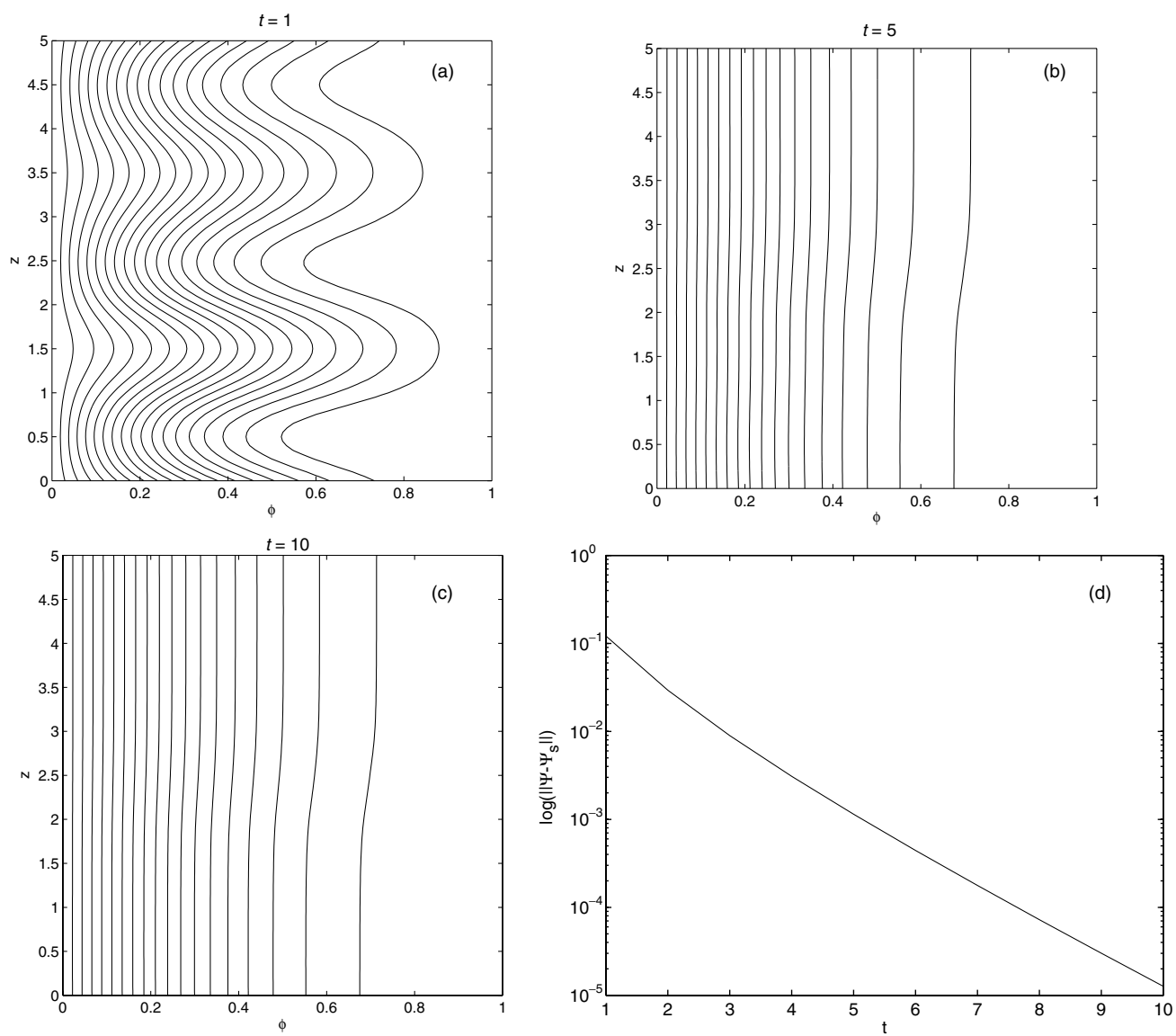

Figure 4. Contours of $\Psi(\phi, \xi, t)$ for the test problem: (a) $t=1$, (b) $t=5$, (c) $t=10$; (d) decay of $\left\|\Psi-\Psi_{\mathrm{s}}\right\|_{L^{2}(\Omega)}(t)$.

are no longer in a Hilbert space setting. Instead the steady model has a solution which lives in the space $W^{1,1+n}(\Omega)$. The solution of the transient model $(2.21)$, is in $H^{1}(\Omega)$ for finite time. Numerically we work in finite-dimensional subspaces, which are anyway in $H^{1}(\Omega)$. For example, in the case of the finite element discretisation we consider basis functions in a subspace of $H^{1}(\Omega)$, which is compactly embedded in $W^{1,1+n}(\Omega)$. Therefore, there is no penalty in taking $\mu_{k, \infty}=0$.

We will consider a varying flow rate, such as would be possible with pressure pulsing at the pump, and investigate the effect of pulsing the flow rate on the displacement. The model is as before, but with boundary condition (4.5) replaced by

$$
\Psi(1, \xi, t)=1+\delta_{p} \sin \omega t .
$$

In the next two subsections we consider two possibilities. First, we consider what happens if we pulsate the flow rate after the mud channel has already been formed. Secondly, what happens when the pulsating flow rate is applied from the beginning of the displacement, i.e. as the interface advances. 


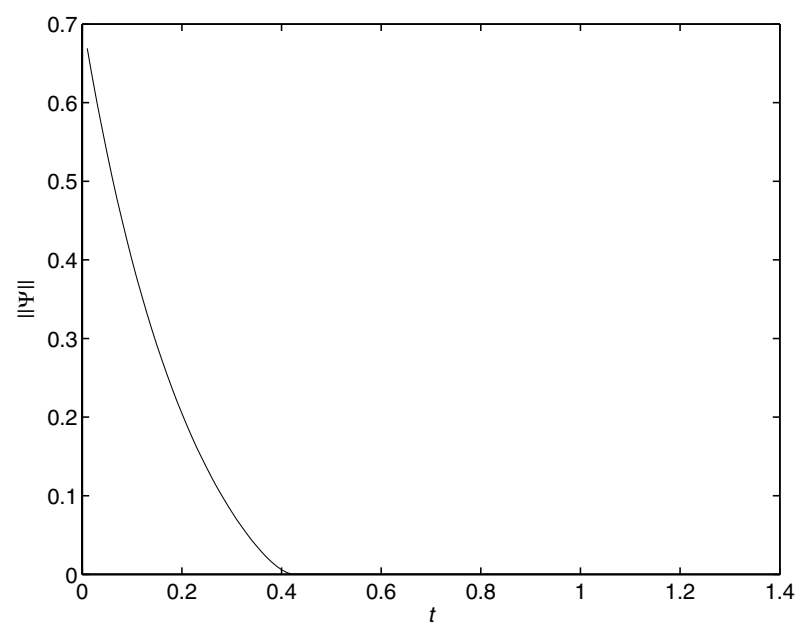

FIGURE 5. Finite-time decay of $\|\Psi\|_{L^{2}(\Omega)}(t) \rightarrow 0$, for zero-imposed flow rate, $Q(t)=0$.

\subsubsection{Pulsation after mud channel forms}

As physical and rheological parameters, we consider the following $\kappa_{1}=0.5, \kappa_{2}=0.4$, $m_{1}=1, m_{2}=1.2, \rho_{1}=1, \rho_{2}=0.9, \tau_{Y, 1}=0.9, \tau_{Y, 2}=0.7$, where $k=1$ corresponds to cement and $k=2$ corresponds to mud. We suppose that the well is vertical and mildly eccentric: $\beta=0, e=0.3$. As time-scale ratio, we take $\epsilon=0.6$ so that viscous and advective timescales are comparable. As oscillation frequency, we adopt $\omega=10$, so that relative to the advective time-scale, the period of oscillation, $T=2 \pi /(\epsilon \omega)$, is also of order unity. Therefore, we expect that transient effects will be fully coupled.

For these parameters, we may verify numerically that there exists a parallel flow of the two fluids, with interface at $\phi=0.8$, such that the displaced fluid is completely static. We take this as an initial condition and simulate the displacement through one full oscillation of the flow rate, at a $20 \%$ amplitude of pulsation, $\delta_{p}=0.2$. We perform the simulation, using both steady and transient models for the stream-function.

Figure 6 shows the effects of the pulsation on the displacement flow, using the steady state velocity model. As we go over the full period of the pulsation the model predicts that the mud channel will remain static for all time. At all times the velocity field appears to remain parallel. As there exists a parallel flow solution at each flow rate during the pulsation, $\Psi_{\mathrm{s}}=\Psi_{\mathrm{s}}(\phi, Q(t))$, this is not surprising. The azimuthal component of velocity is therefore always zero and the interface is not disturbed.

In Figure 7 we show the results of the same simulation, but using the transient model. As we go over the full period of the pulsation the mud channel slowly begins to yield until it is fully moving, then goes back to the static mud channel after the pulsation period is over. Thus, in each pulsation the mud channel will yield, move up the narrow side for a short period of time and stop again. As with the steady state velocity model the interface remains parallel. An interesting behaviour is that it seems that the interface is stable and unperturbed. In a companion paper we study interfacial stabilities and find that the interface remains stable in a steady flow if the narrow side channel remains 


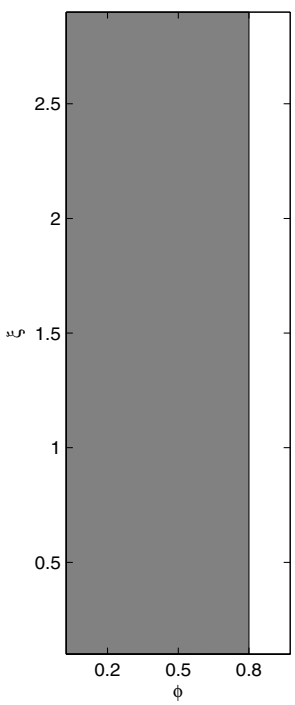

(a)

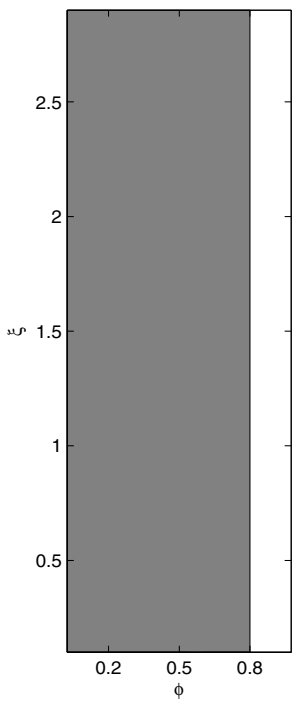

(e)

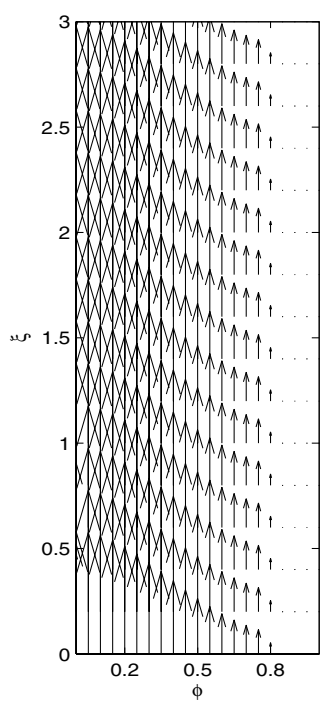

(b)

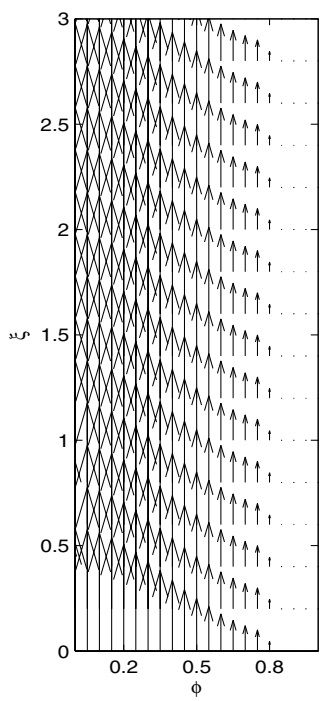

(f)

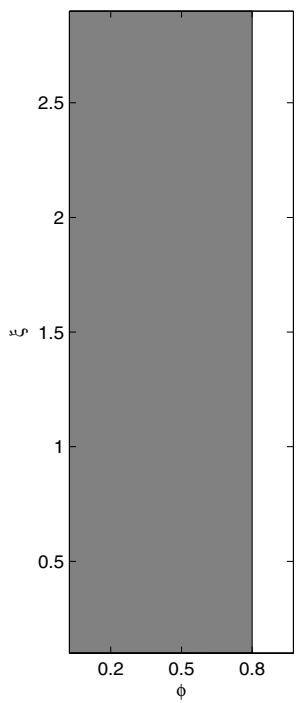

(c)

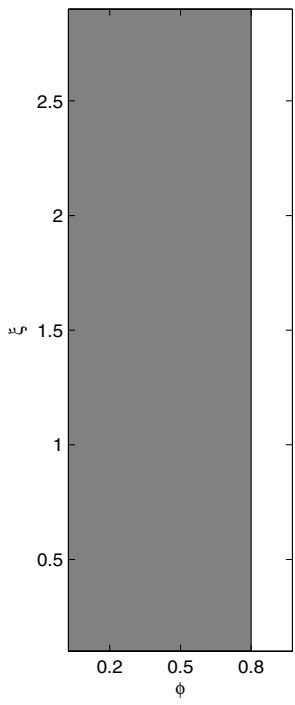

(g)

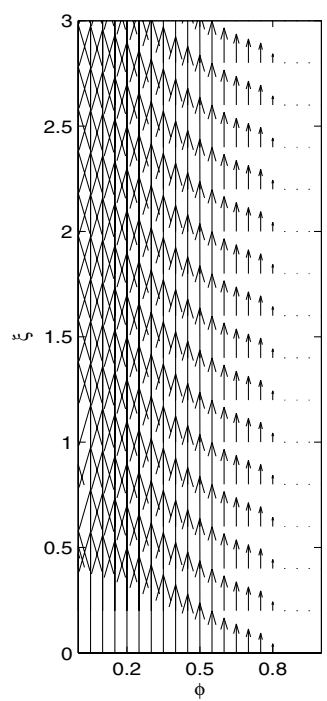

(d)

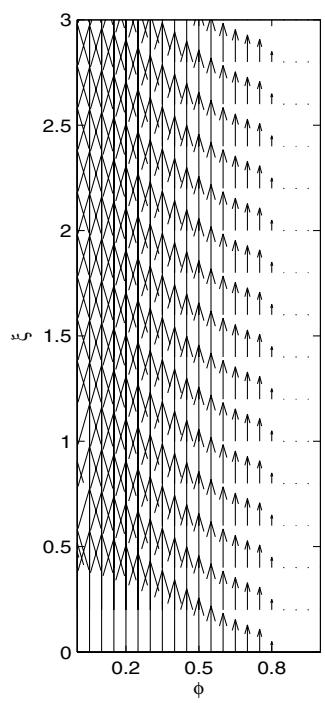

(h)

FIGURE 6. Displacement flow in an eccentric annulus with static mud channel and pseudo steady velocity model. Period $T=2 \pi / \omega, \omega=10$, and $\delta_{\mathrm{p}}=0.2$. Interface position and velocity field for times: (a)-(b) $T / 4$. (c)-(d) $T / 2$. (e)-(f) $3 T / 4$. (g)-(h) $T$. Physical and rheological parameters: $\kappa_{1}=0.5$, $\kappa_{2}=0.4, m_{1}=1, m_{2}=1.2, \rho_{1}=1, \rho_{2}=0.9, \tau_{Y, 1}=0.9, \tau_{Y, 2}=0.7 e=0.3, \beta=0$. Mud-white, cement-grey.

unyielded. Although the mud channel does move on each cycle, it may be that the time period when the fluid is yielded is not long enough for instabilities to grow, or that for these parameters the flow is in fact stable.

To summarise, our results indicate that if the static mud channel is allowed to form, it is unlikely to become unstable and be removed via pulsation. We turn therefore to the study of the effects of pulsation during the displacement itself. 


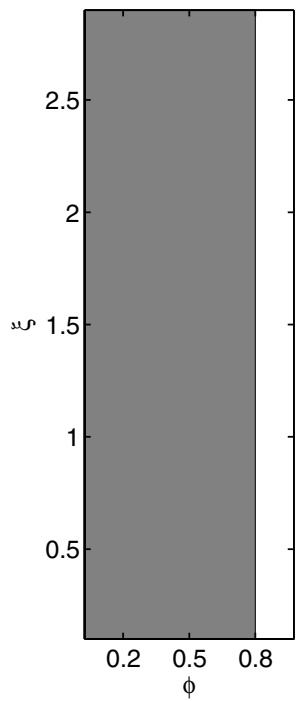

(a)

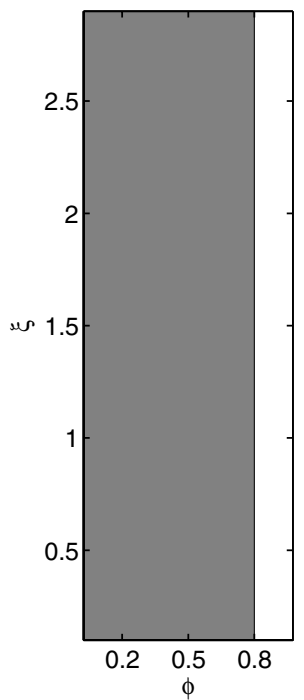

(e)

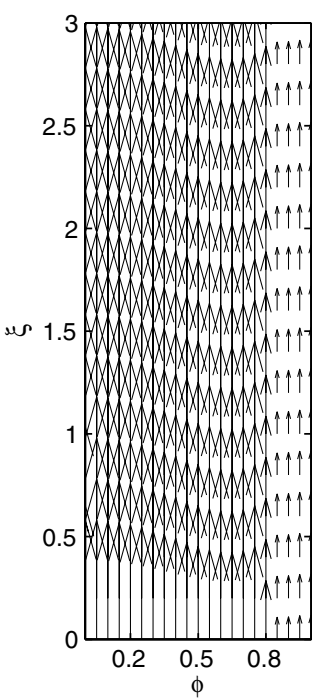

(b)

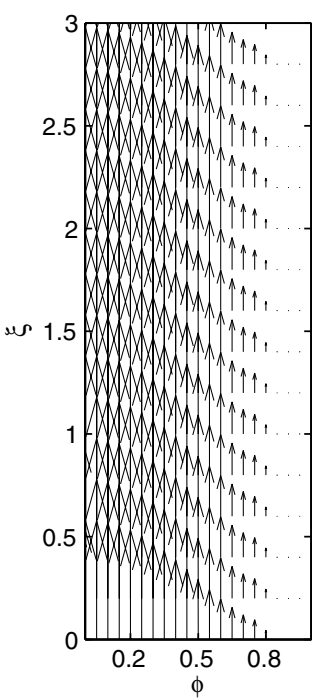

(f)

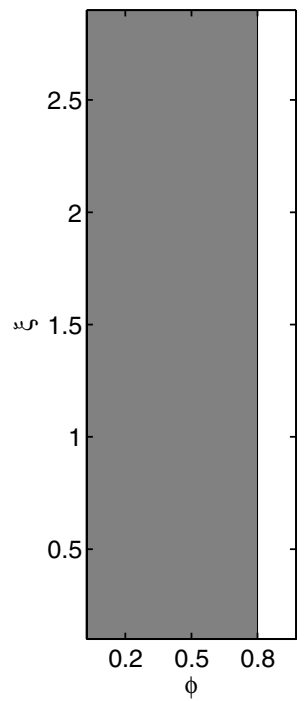

(c)

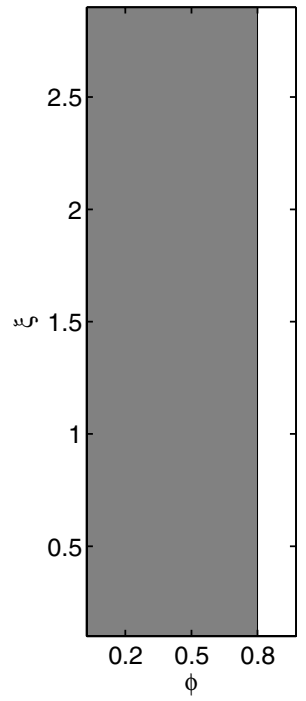

(g)

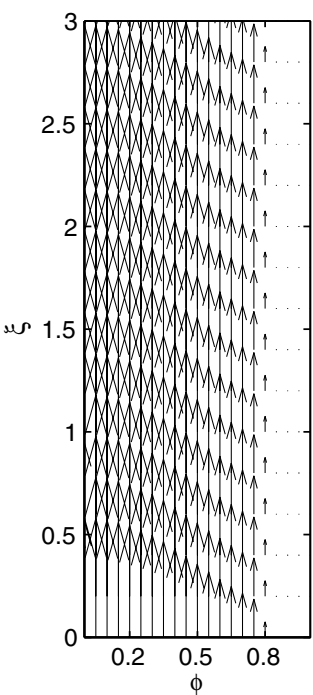

(d)

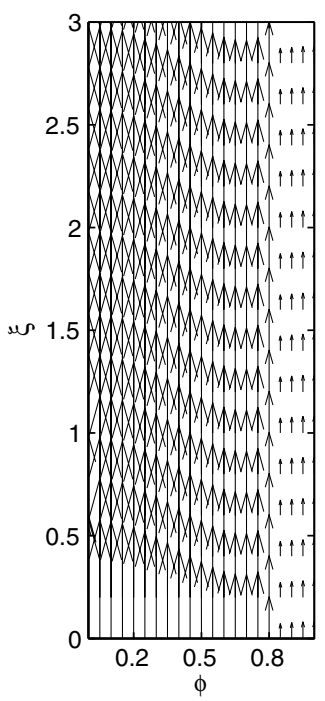

(h)

FIGURE 7. Displacement flow in eccentric annulus with a static mud (white) channel using the transient velocity model. Period $T=2 \pi / \omega \epsilon, \omega=10, \delta_{p}=0.2$, and $\epsilon=0.6$. Interface position and velocity field for times: (a)-(b) $T / 4$. (c)-(d) $T / 2$. (e)-(f) $3 T / 4$. (g)-(h) $T$. Physical and rheological parameters: $\kappa_{1}=0.5, \kappa_{2}=0.4, m_{1}=1, m_{2}=1.2, \rho_{1}=1, \rho_{2}=0.9, \tau_{Y, 1}=0.9, \tau_{Y, 2}=0.7 e=0.3, \beta=0$. Mud-white, cement-grey.

\subsubsection{Pulsation as the displacement front passes}

We simulate the effects of pulsation on a displacement front that is initially perpendicular to the annulus axis, for $10 \%$ and $20 \%$ pulsation amplitudes. Figures 8 and 9 show the width of the mud channel with pulsation amplitudes $\delta_{p}=0.1$ and $\delta_{p}=0.2$ respectively, after 


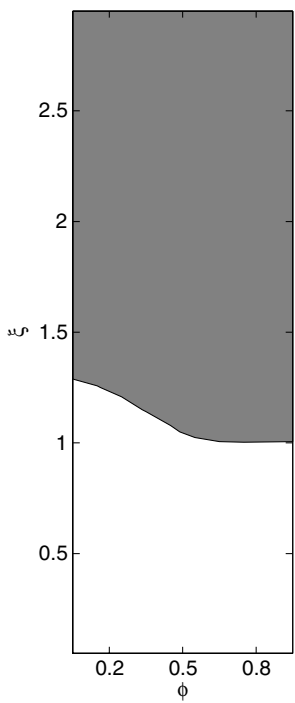

(a)

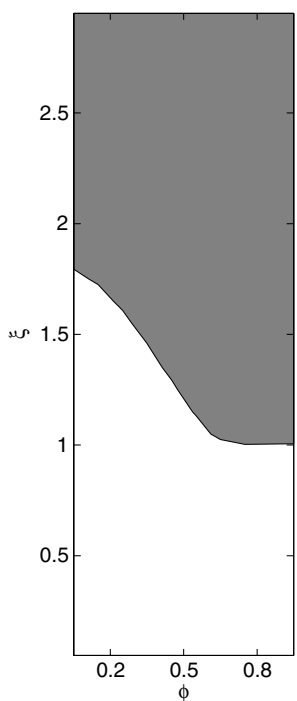

(e)

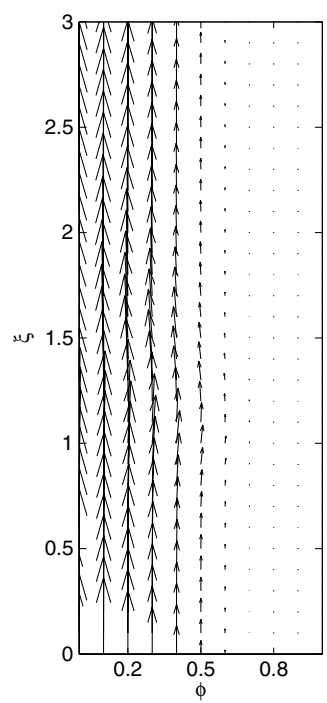

(b)

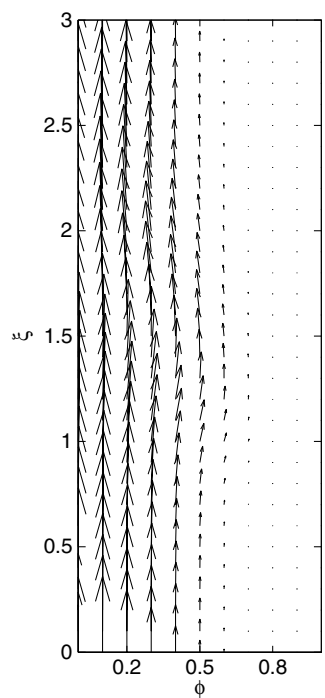

(f)

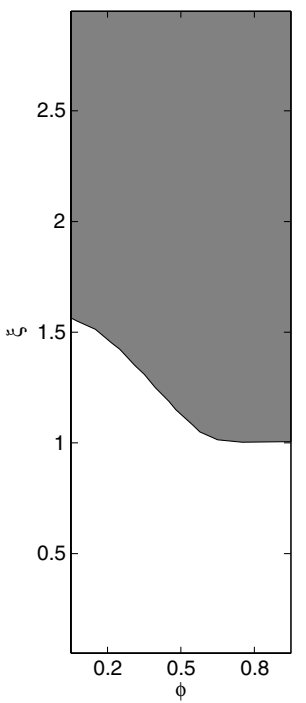

(c)

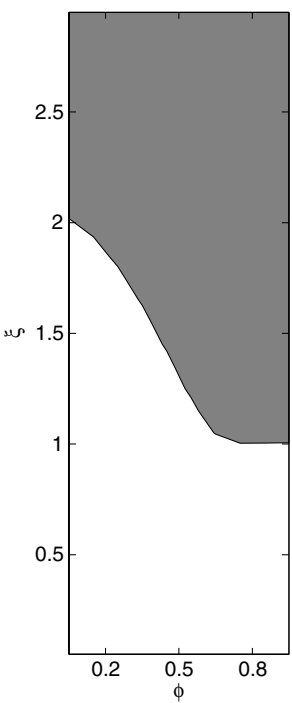

(g)

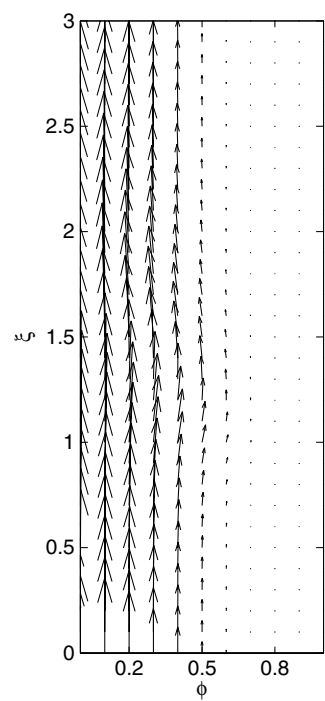

(d)

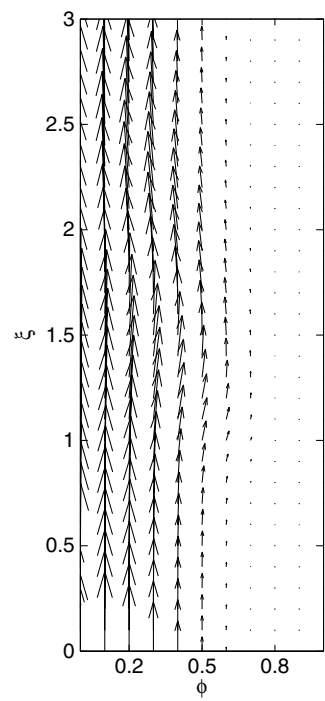

(h)

FIGURE 8. Displacement flow in eccentric annulus, interface propagation. Mud channel formation. Pseudo-steady velocity model. Period $T=2 \pi / \omega, \omega=10$, and $\delta_{\mathrm{p}}=0.1$. Interface position and velocity field for times: (a)-(b) $T / 4$. (c)-(d) $T / 2$. (e)-(f) $3 T / 4$. (g)-(h) $T$. Physical and rheological parameters: $\kappa_{1}=0.5, \kappa_{2}=0.4, m_{1}=1, m_{2}=1.2, \rho_{1}=1, \rho_{2}=0.9, \tau_{Y, 1}=0.9, \tau_{Y, 2}=0.7 e=0.8, \beta=0$. Cement-white, mud-grey.

solving the pseudo-steady model. There is no clear evidence of a change in the position of the interface on the narrow side. Figures 10 and 11 show the effects of a pulsating flow rate on the transient model with amplitudes, $\delta_{\mathrm{p}}=0.1$ and $\delta_{\mathrm{p}}=0.2$, respectively. The narrow-side fluids move more than with the pseudo-steady model. In Figure 12 we show a close-up of the velocity profiles at $\xi=1.5$, comparing directly between pseudo-steady and transient models. Even though the velocities are zero far upstream and downstream 


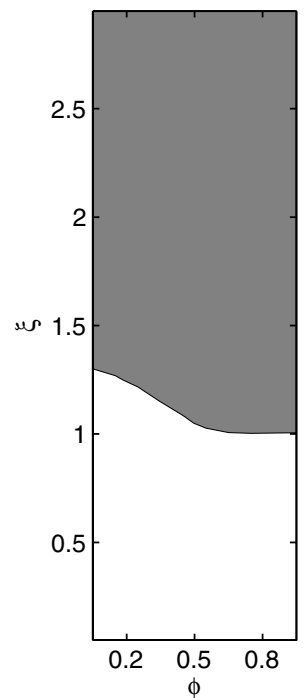

(a)

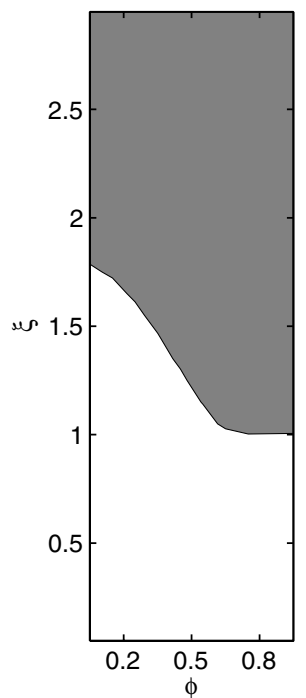

(e)

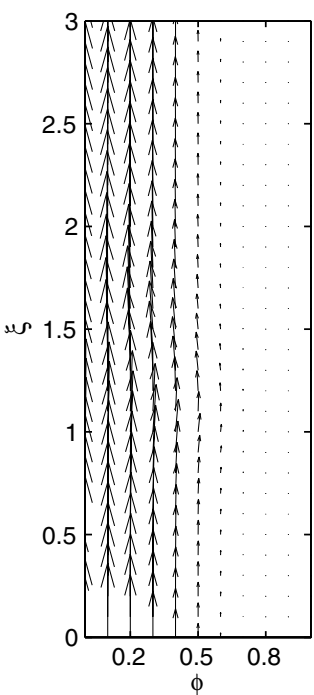

(b)

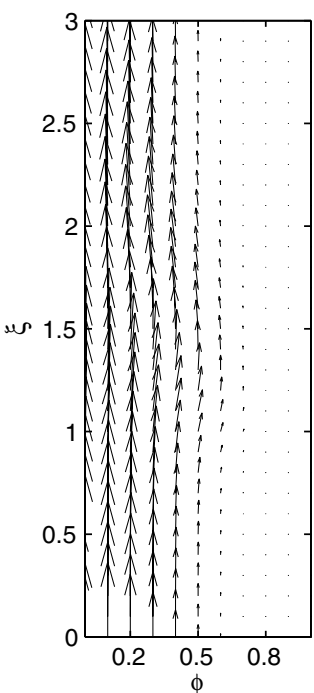

(f)

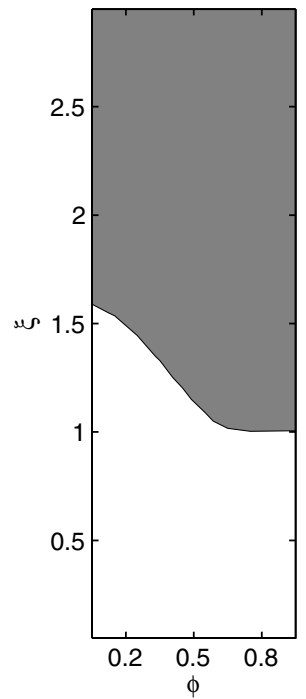

(c)

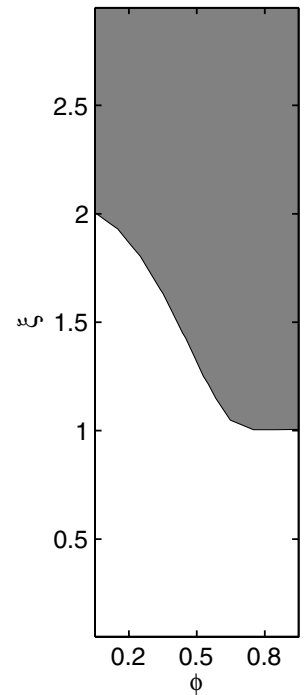

(g)

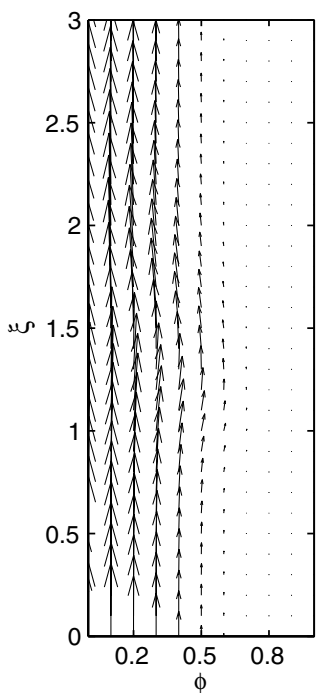

(d)

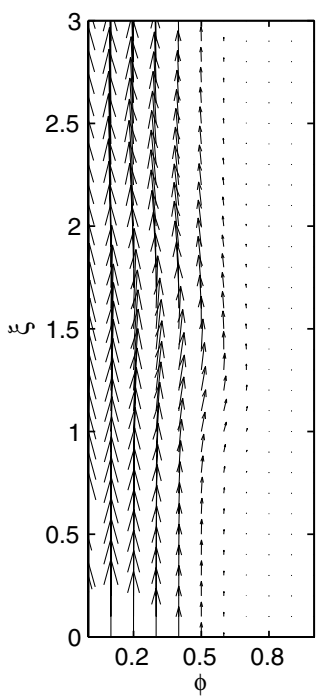

(h)

FIgURE 9. Displacement flow in eccentric annulus, interface propagation. Mud channel formation. Pseudo-steady velocity model. Period $T=2 \pi / \omega, \omega=10$, and $\delta_{\mathrm{p}}=0.2$. Interface position and velocity field for times: (a)-(b) $T / 4$. (c)-(d) $T / 2$. (e)-(f) $3 T / 4$. (g)-(h) $T$. Physical and rheological parameters: $\kappa_{1}=0.5, \kappa_{2}=0.4, m_{1}=1, m_{2}=1.2, \rho_{1}=1, \rho_{2}=0.9, \tau_{Y, 1}=0.9, \tau_{Y, 2}=0.7, e=0.8, \beta=0$. Cement-white, mud-grey.

of the interface, the interface does move, via a burrowing motion (see also [16]), in which the fluids are locally yielded close to the interface. This yielded region advances slowly with the interface along the annulus. As we increase the magnitude of the pulsation, this yielding motion expands further towards the narrow side of the annulus, and therefore a decrease of the width of the mud channel is achieved. 


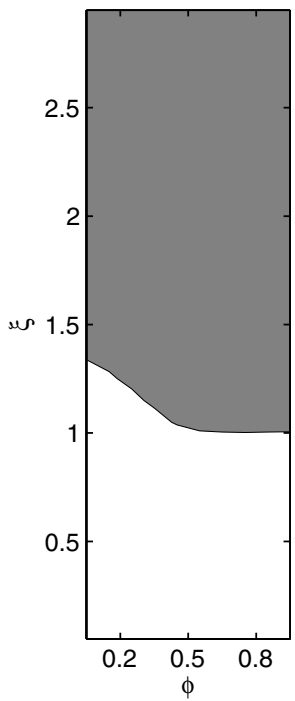

(a)

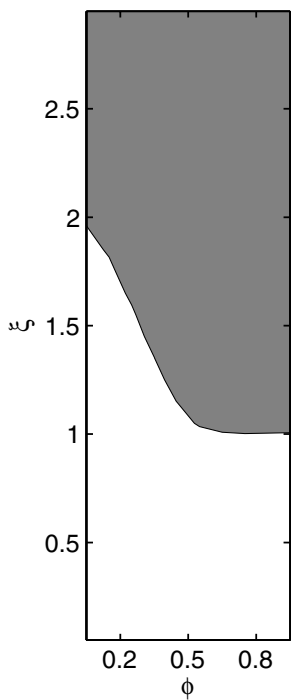

(e)

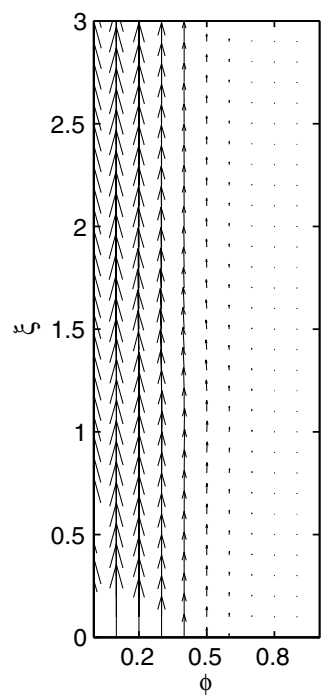

(b)

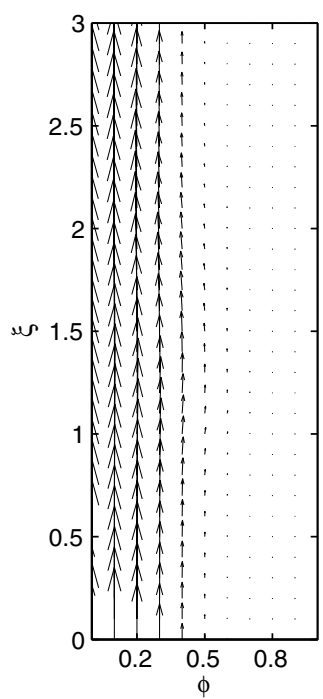

(f)

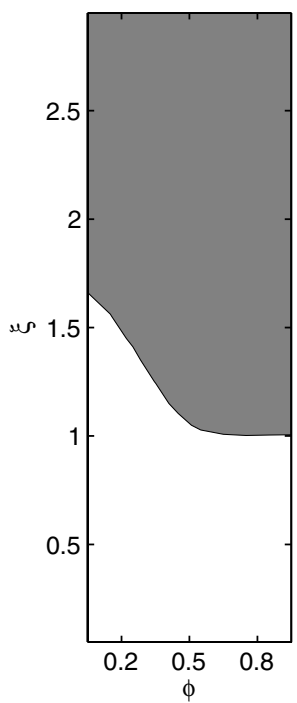

(c)

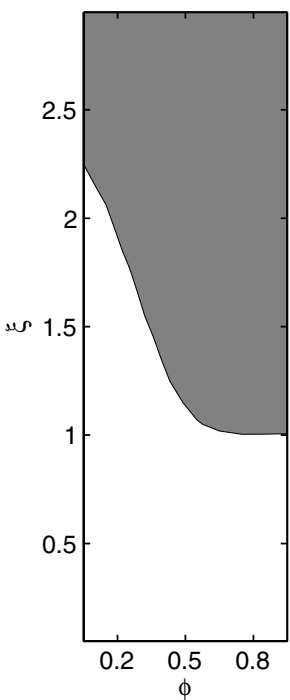

(g)

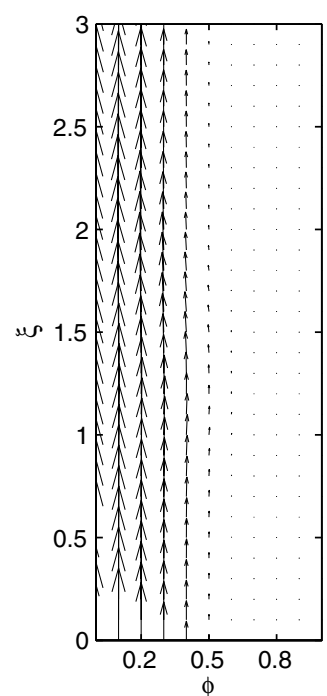

(d)

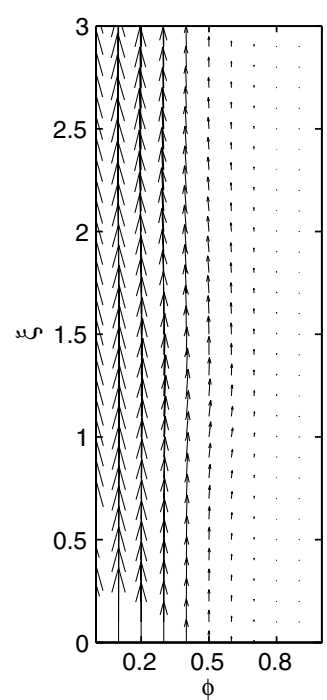

(h)

FiguRE 10. Displacement flow in eccentric annulus, interface propagation. Mud channel formation. Transient velocity model. Period $T=2 \pi / \omega \epsilon, \omega=10, \delta_{\mathrm{p}}=0.1$, and $\epsilon=0.6$. Interface position and velocity field for times: (a)-(b) $T / 4$. (c)-(d) $T / 2$. (e)-(f) $3 T / 4$. (g)-(h) $T$. Physical and rheological parameters: $\kappa_{1}=0.5, \kappa_{2}=0.4, m_{1}=1, m_{2}=1.2, \rho_{1}=1, \rho_{2}=0.9, \tau_{Y, 1}=0.9, \tau_{Y, 2}=0.7, e=0.8, \beta=0$. Cement-white, mud-grey.

As a conclusion, the transient model (4.1)-(4.7) appears to lead to a reduction of the mud channel. Therefore, pulsation of the flow rate at the beginning of the displacement might be used as a tool to reduce mud channels along the annuli. 


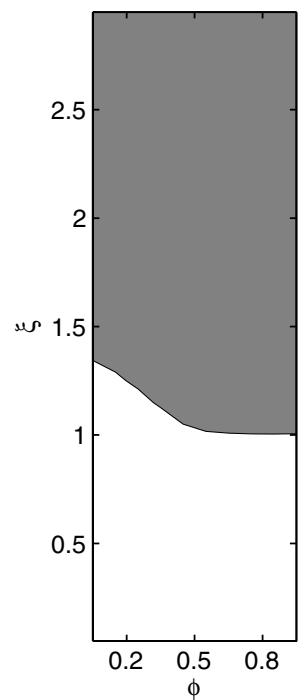

(a)

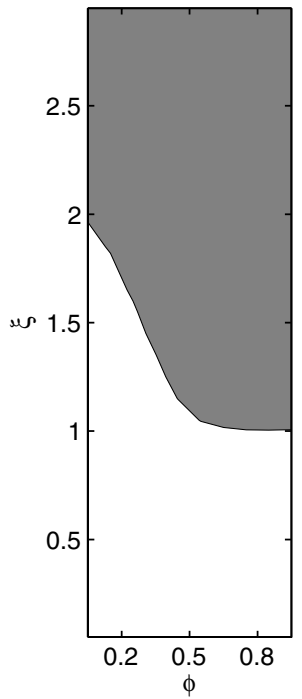

(e)

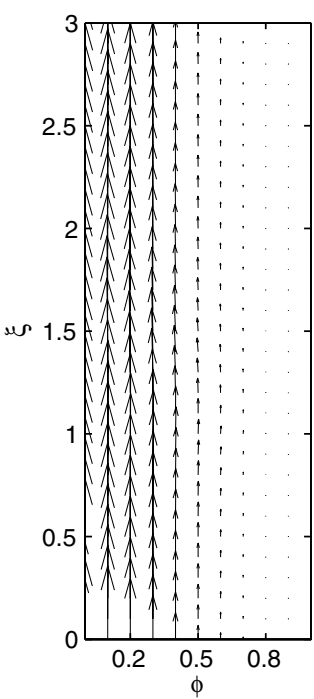

(b)

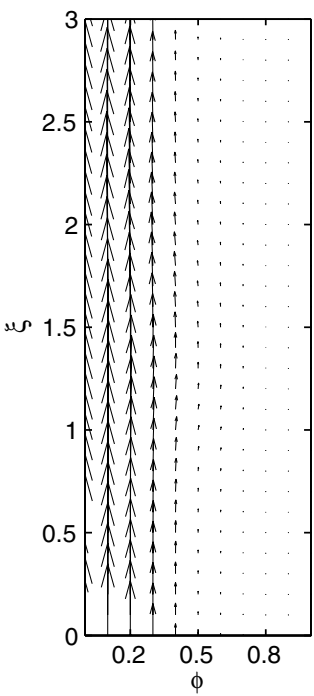

(f)

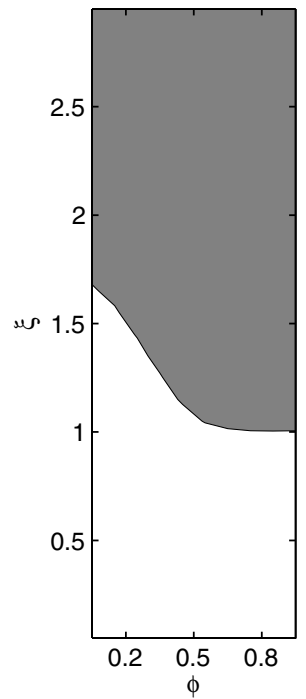

(c)

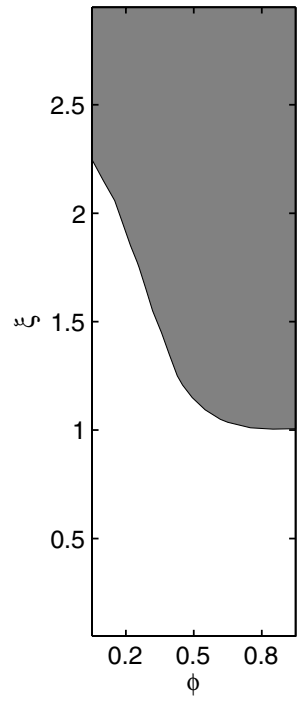

(g)

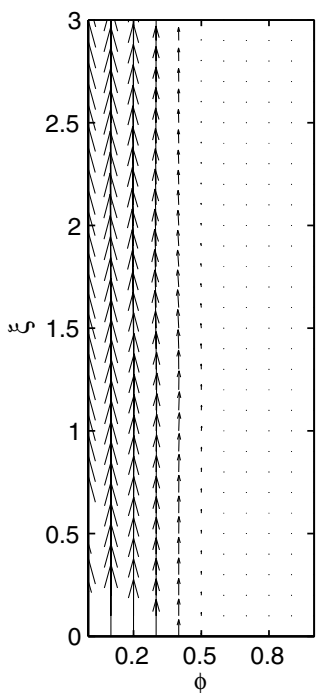

(d)

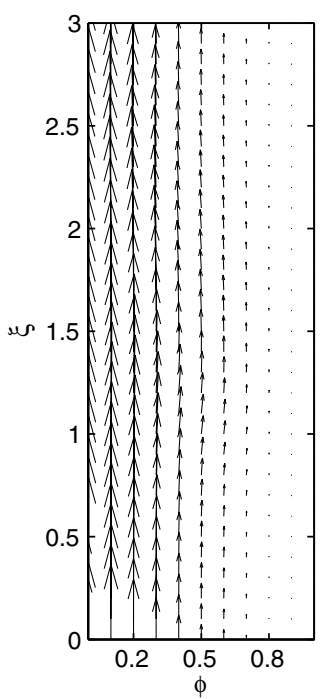

(h)

FIGURE 11. Displacement flow in eccentric annulus, interface propagation. Mud channel formation. Transient velocity model. Period $T=2 \pi / \omega \epsilon, \omega=10, \delta_{\mathrm{p}}=0.2$, and $\epsilon=0.6$. Interface position and velocity field for times: (a)-(b) $T / 4$. (c)-(d) $T / 2$. (e)-(f) $3 T / 4$. (g)-(h) $T$. Physical and rheological parameters: $\kappa_{1}=0.5, \kappa_{2}=0.4, m_{1}=1, m_{2}=1.2, \rho_{1}=1, \rho_{2}=0.9, \tau_{Y, 1}=0.9, \tau_{Y, 2}=0.7, e=0.8, \beta=0$. Cement-white, mud-grey.

\section{Discussion}

In this paper we have developed the mathematical foundations for a transient version of the displacement model in [3,15-17]. The basic building block of this type of model is 


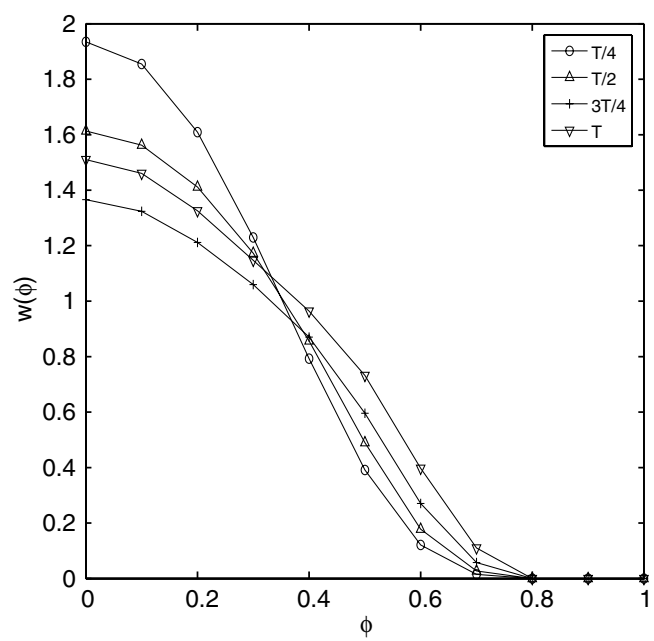

(a)

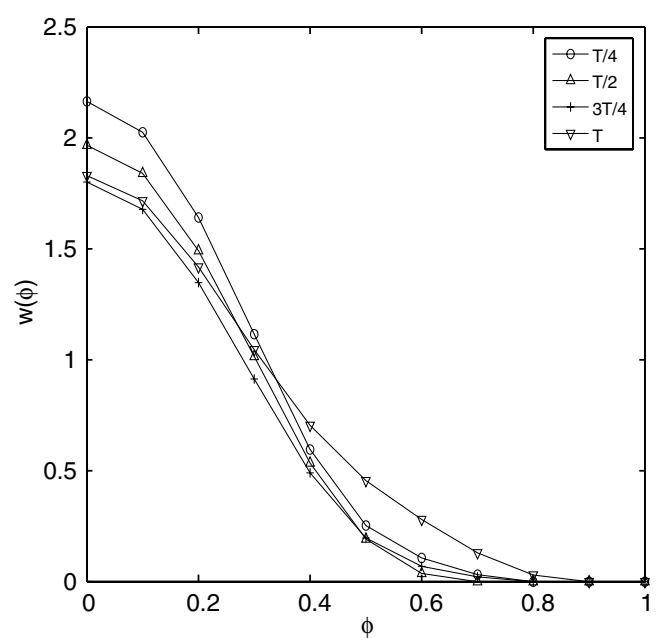

(c)

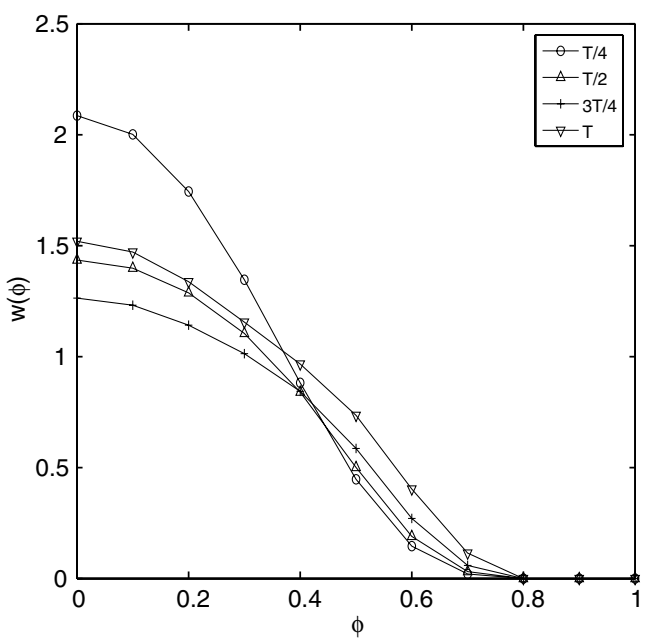

(b)

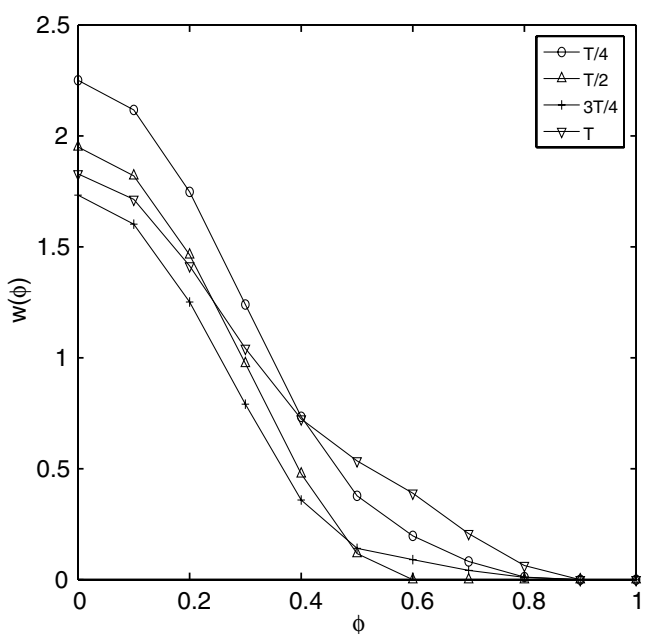

(d)

FIGURE 12. Velocity profile at $\xi=1.5$ : (a)-(b) Pseudo-steady model, $\delta_{\mathrm{p}}=0.1,0.2$, respectively. (c)-(d) Transient model, $\delta_{\mathrm{p}}=0.1,0.2$, respectively.

a Hele-Shaw model of a yield stress fluid. The porous media analogue of such flows is a non-linear Darcy flow with limiting pressure gradients. Such flows have been studied extensively by Entov and co-workers (see e.g. [2] and references therein). In this context, the annular eccentricity corresponds to an anisotropic variation in the permeability and hence flow law. What is perhaps interesting is that the porous media analogy concerns primarily steady flows. For porous media flows, transient terms enter via the mass conservation equation, as a pressure (density) time derivative for weakly compressible fluids. Here the transient terms are accelerations.

Our model is essentially a hydraulics model, but in two dimensions. Inclusion of the acceleration terms allows the pressure gradient to locally relax from the frictional 
pressure gradient of a steady laminar flow between parallel plates, i.e. the Hele-Shaw approximation. This relaxation allows for different dynamics from those with the pseudosteady model, as we have seen in Section 4.3. In our companion paper we will also see that the transient model admits interfacial instabilities whereas the pseudo-steady model does not.

The approximations that we have made are typical of those made in hydraulics, i.e. averaging across the annular gap, interchanging derivative and averaging operations. A natural question to ask is whether the non-linear inertial terms could have been included within this formulation? This is of course, possible, with the assumptions on being able to approximate the averages of the spatial derivatives of the velocity with the spatial derivatives of the averages. Equation (2.21) is replaced by

$$
\begin{aligned}
\nabla \cdot\left(\frac{\rho}{H}\left[\nabla \Psi_{t}+\tilde{\operatorname{Re}} \mathbf{N}(\Psi)\right]\right) & =-\nabla \cdot[\mathbf{S}+\mathbf{f}], \\
\mathbf{N}(\Psi) & =\left(\Psi_{\phi} \Psi_{\phi \xi}-\Psi_{\xi} \Psi_{\phi \phi}, \Psi_{\phi} \Psi_{\xi \xi}-\Psi_{\xi} \Psi_{\phi \xi}\right),
\end{aligned}
$$

where $\tilde{R e}$ is an appropriately defined Reynolds number. The term $\nabla \cdot \mathbf{S}$ is essentially elliptic, second order in $\Psi$, whereas the new terms in $\nabla \cdot\left[\frac{\rho}{H} \mathbf{N}(\Psi)\right]$ will contain third-order spatial derivatives. It is not clear how (5.1) is changed by this. Intuitively we expect that for $\tilde{R e} \ll 1$ we have a unique solution, perturbed from that we have considered and that for larger $\tilde{R e}$ this uniqueness will be lost. However, we have not developed any analysis of this equation.

The numerical method we have used is one that is commonly used for transient flows of visco-plastic fluids, but in the Navier-Stokes context. Here the yielding behaviour is preserved in the gap-averaged model, but the time-derivative operates on $E[\Psi]$ which is elliptic. The visco-plastic fluids analogy would be to study an unsteady one-dimensional duct flow, but with the time derivative replaced by (minus) the time derivative of the Laplacian. It is of interest to note that recently this method has been advocated as a way to compute steady-state visco-plastic duct flows using the augmented Lagrangian method, but giving convergence of the steady-state velocity approximations in $H^{1}$ rather than in $L^{2}$ [12]. Of course, this is a purely numerical trick, with no physical basis. In contrast, here we have the physical motivation for a very similar numerical problem.

Finally a comment on the effects of flow pulsation. We have shown in Section 4.3 that pulsation using a transient model tends to make the fluids more mobile on the narrow side of the annulus. Although the effects might not seem dramatic, it is important to understand that unremoved drilling mud channels are a serious industrial problem, and any help towards mobilising them is significant. For example, mobile drilling mud may be contaminated by chemical additives on a faster time scale than static mud.

\section{Acknowledgements}

This research has been carried out at the University of British Columbia, supported financially by Schlumberger and NSERC through CRD project 245434; MM-G \& IF. Part of the research has been carried out during a study visit at UBC in August 2004 during which O.S. was supported as a visiting professor by the Pacific Institute for the Mathematical Sciences. 


\section{References}

[1] Andreu, F., Ballester, C., Caselles, V. \& Mazón, J. M. (2001) The Dirichlet problem for the total variation flow. J. Funct. Analy. 180, 347-403.

[2] Barenblatt, G. I., Entov, V. M. \& Ryzhik, V. M. (1990) Theory of Fluid Flows Through Natural Rocks. Kluwer Academic Publishers, New York.

[3] Bittleston, S. H. Ferguson, J. \& FrigaArd, I. A. (2002) Mud removal and cement placement during primary cementing of an oil well; laminar non-Newtonian displacements in an eccentric annular Hele-Shaw cell. J. Eng. Math. 43, 229-253.

[4] Dacorogna, B. (1989) Direct Methods in the Calculus of Variations. AMS Vol. Springer-Verlag.

[5] Dustrehoft, D., Wilson, G. \& Newman, K. (2002) Field Study on the Use of Cement Pulsation to Control Gas Migration. Society of Petroleum Engineers paper number SPE 75689.

[6] Ekeland, I. \& TÉmam, R. (1999) Convex Analysis and Variational Problems. SIAM.

[7] Evans, L. C. (1998) Partial Differential Equations. American Mathematical Society.

[8] Fortin, M. \& Glowinski, R. (1983) Augmented Lagrangian Methods. North-Holland.

[9] Galdi, P. (1991) An Introduction to the Mathematical Theory of the Navier Stokes Equations, Part 1. Springer-Verlag, 463 pp.

[10] Gilbarg, D. \& Trudinger, N. S. (1983) Elliptic Partial Differential Equations of Second Order. Springer-Verlag, New York.

[11] Glowinski, R. (1983) Augmented Lagrangian Methods: Applications to the Numerical Solution of Boundary-Value Problems. North-Holland.

[12] He, J. W. \& Glowinski, R. (2000) Steady Bingham fluid flow in cylindrical pipes: a time dependent approach to the iterative solution. Numer. Linear Algebra Appl. 7(6), 381-428.

[13] Moyers-González, M. A. (2006) Transient Effects in Oilfield Cementing flows. Ph.D. thesis, University of British Columbia.

[14] Moyers-González, M. A. \& FrigaArd, I. A. (2006) Kinematic instabilities in two-layer eccentric annular flows, part 1: Newtonian fluids. J. Eng. Math., October, accepted for publication.

[15] Pelipenko, S. \& Frigand, I. A. (2004) On steady state displacements in primary cementing of an oil well. J. Eng. Math. 46(1), 1-26.

[16] Pelipenko, S. \& FrigaArd, I. A. (2004) Two-dimensional computational simulation of eccentric annular cementing displacements. IMA J. Appl. Math. 69, 557-583.

[17] Pelipenko, S. \& FrigaArd, I. A. (2004) Visco-plastic fluid displacements innear-vertical eccentric annuli: lubrication modelling. J. Fluid Mech. 520, 343-377.

[18] Tehrani, A., Bittleston, S. H. \& Long, P. J. (1993) Flow instabilities during annular displacement of one non-Newtonian fluid by another. Exp. Fluids 14, 246-256.

[19] ZALESAK, S. T. (2001) Fully multidimensional flux-corrected transport algorithms for fluids. J. Non-Newt. Fluid Mech. 100, 49-76. 